\title{
SAND2000-0934J
}

\section{Adhesion hysteresis of silane coated microcantilevers}

\author{
M. P. de Boer ${ }^{a}$, J. A. Knapp ${ }^{b}$, and T. A. Michalske ${ }^{c}$ \\ Sandia National Laboratories, Albuquerque, NM 87185 \\ ${ }^{2}$ Dept. 1725, Intelligent Micromachine Technology, www.mdl.sandia.gov/Micromachine \\ ${ }^{\mathrm{b}}$ Dept. 1111, Radiation Solid Interactions and Processing \\ ${ }^{c}$ Dept. 1115, Nanomolecular Materials and Interfaces
}

U. Srinivasan and R. Maboudian

Dept. of Chemical Engineering, Univ. of CA, Berkeley, CA 94720

Abstract

We have developed a new experimental approach for measuring hysteresis in the adhesion between micromachined surfaces. By accurately modeling the deformations in cantilever beams that are subject to combined interfacial adhesion and applied electrostatic forces, we determine adhesion energies for advancing and receding contacts. We draw on this new method to examine adhesion hysteresis for silane coated micromachined structures and found significant hysteresis for surfaces that were exposed to high relative humidity (RH) conditions. Atomic force microscopy studies of these surfaces showed spontaneous formation of agglomerates that we interpreted as silanes that have irreversibly transformed from uniform surface layers at low RH to isolated vesicles at high RH. We used contact deformation models to show that the compliance of these vesicles could reasonably account for the adhesion hysteresis that develops at high RH as the surfaces are forced into contact by an externally applied load. 
de Boer et al., to be submitted to Acta Mater., Feb. '00

\section{Introduction}

Adhesion and friction are significant tribological concerns in addressing the reliability of Microelectromechanical (MEMS) devices [1,2]. This is because the surface to volume ratio of structures grows as dimensions shrink. The structural members in MEMS are only a few microns thick and a few micrometers ( $\mu \mathrm{ms})$ apart, and are often constructed of polycrystalline silicon (polysilicon) that exhibits nanometer scale root mean square (rms) roughness. The surface properties of MEMS devices are strongly related to their processing, as well as their environmental exposure. For example, polysilicon roughness depends on its deposition temperature [3]. Likewise, the surface energy of the polysilicon depends on the final surface treatment process. If supercritical drying [4] is performed to release the parts. the surfaces are high energy and hydrophilic. The surfaces can instead be rendered hydrophobic by applying monolayer lubrication layers [5]. Contamination or deposition kinetics in the surface treatment process can result in non-uniform films [6-9], and may affect adhesion on a local scale. Therefore, it is important to develop in-situ tribology techniques on as-fabricated MEMS parts. These will eliminate the question of whether measured results from macroscopic tribological tools reflect real properties of MEMS structures. Furthermore, issues of coating stability, aging and wear, important in micromachined products [6], can be directly addressed.

Knowledge of adhesion hysteresis values is valuable in developing a fundamental understanding of tribology. The surface forces apparatus (SFA) [10] is commonly used to study adhesion and friction of monolayer coatings. A link often made between these properties is adhesion hysteresis, the difference between the adhesion energy measured when two surfaces are brought into contact versus when they are being separated [11]. The connection is that the static friction coefficient is related to the adhesion values of propagating cracks, while the dynamic friction coefficient is related to adhesion hysteresis values. Friction experiments on sliding surfaces of micrometer scale roughness have also led to the notion that the age of contact is critical in determining coefficients of friction [12], suggesting that hysteresis is also an important key to understanding friction laws of rough surfaces.

In this paper, we develop an in-situ technique for measuring adhesion hysteresis of an asfabricated MEMS test structure, and report experimental values of hysteresis at low and high humidity levels for a film coated with perfluorotrichlorosilane $\left(\mathrm{C}_{8} \mathrm{~F}_{17} \mathrm{C}_{2} \mathrm{H}_{4} \mathrm{SiCl}\right.$. FDTS), a monolayer lubricant. The work establishes an important element in the MEMS tribological 


\section{DISCLAIMER}

This report was prepared as an account of work sponsored by an agency of the United States Government. Neither the United States Government nor any agency thereof, nor any of their employees, make any warranty, express or implied, or assumes any legal liability or responsibility for the accuracy, completeness, or usefulness of any information, apparatus, product, or process disclosed, or represents that its use would not infringe privately owned rights. Reference herein to any specific commercial product, process, or service by trade name, trademark, manufacturer, or otherwise does not necessarily constitute or imply its endorsement, recommendation, or favoring by the United States Government or any agency thereof. The views and opinions of authors expressed herein do not necessarily state or reflect those of the United States Government or any agency thereof. 


\section{DISCLAIMER}

Portions of this document may be illegible in electronic image products. Images are produced from the best available original document. 
toolbox we are developing. The hysteresis measurements also allow us to determine the local uniformity of adhesion, as well as the effect of contact history on adhesion in MEMS.

Furthermore, they are accomplished in a small area $\left(15 \times 2000\right.$ micron $^{2}$ for each measurement), important because of the expense of real-estate on a MEMS wafer.

Mechanics for measurement of adhesion in the presence of an externally applied load 1) Analytical equations

In our experiments we employ cantilever beams to study the adhesion due to interfacial forces. Adhesion hysteresis experiments require application of a load to heal and propagate cracks. We provide an externally applied load by means of electrostatic forces to move the crack, as in Fig. 1. From an interfacial fracture mechanics perspective, the non-adhered region from the cantilever support post to the point of attachment is considered to be a crack of length $s$. Note that the beam and landing pad are electrically grounded, such that only adhesive forces and no electrostatic forces operate in the contact zone $d$. Voltage $V_{\text {pad }}$ is applied to the actuation pad far away from the crack tip. Increasing $V_{\text {pad }}$ heals the cracks, while decreasing $V_{\text {pad }}$ propagates the cracks, corresponding to advancing and receding contacts.

We first develop an analytical model to gain insight into the mechanics of this geometry. Consider, as in Fig. 2, a cantilever beam of length $L$, thickness $t$ and height $h$ adhered to the substrate over a distance $d=L-s$, and subject to an externally applied uniform distributed load $q$. For $q=0$, with the Johnson, Kendall and Roberts (JKR) assumption [13] (adhesion forces only in the contact zone over the attachment length $d$ as represented by the cross-hatches), assuming free interfacial slip, an elastically rigid support post and a rigid substrate, its deflection profile is [14]

$$
v(x)=h\left[3\left(\frac{x}{s}\right)^{2}-2\left(\frac{x}{s}\right)^{3}\right]
$$

This applies for S-shaped beams such that the slope of the beam at the point of contact with the substrate is zero [14]. The effective energy of adhesion $\Gamma\left(\mathrm{J} / \mathrm{m}^{2}\right)$ of the beam to the substrate can be measured when the energy release rate in the absence of an applied external load, $G_{o}$, has reached a static equilibrium. Then 
$G_{o}=\frac{18 D h^{2}}{s^{4}}=\Gamma$

where $D=E I / w=E t^{3} / 12$ ( $E$ is Young's Modulus, $w$ is the width of the beam and $I=w t^{3} / 12$ is the moment of inertia of the beam, and $t$ is the thickness).

If now we apply $q$ over the interval $0 \leq x \leq a$ as in Fig. 2, the crack length $s$ will tend to decrease. To determine adhesion in the presence of an external load $q$, we must first calculate the deflection curves of the beams for a given crack length. Knowing this, we can then find the energy release rate $G_{q}$ in the presence of the applied load $q$. The important results are outlined next, and the details are given in the appendix.

The deflection curves for $v_{1}(x)$ over the intervals $0 \leq x \leq a$ per Eq. (Al) (see the appendix) and for $v_{2}(x)$ over the interval $a \leq x \leq L$ per Eq. (A2) are shown in Fig. 3 for a fixed value of $s$ and decreasing $\Gamma\left(\mathrm{J} / \mathrm{m}^{2}\right)$. Here we choose $a=60 \mu \mathrm{m}, t=h=2 \mu \mathrm{m}$, Young's Modulus $E=160 \mathrm{GPa}$ [15], hold $s=1000 \mu \mathrm{m}$ constant and increase $q$. The values for $E, t$ and $h$ are typical for micromachined polycrystalline silicon (polysilicon). (Note that downwards corresponds to a positive deflection according to standard beam theory conventions). In Fig. 3 as $q$ increases, the deflections increase and the adhesion $\Gamma$ decreases because $s$ is held constant. The value for $q_{\max }$ corresponds to $\Gamma=0$, as discussed below.

Per Eq. (A12), the result for $G_{q}$ is

$$
G_{q}=G_{o}+\frac{q^{2} a^{6}(4 s-3 a)^{2}}{288 s^{4} D}-\frac{q a^{3} h}{2 s^{4}}(4 s-3 a)
$$

where $D=E t^{3} / 12$ is the beam bending stiffness. Inserting the numbers above, $G_{o}=7.68 \mu \mathrm{J} / \mathrm{m}^{2}$ (i.e., microjoules per square meter) from Eq. (2), while $G_{q}=1.67 \mu \mathrm{J} / \mathrm{m}^{2}$ with $q=10 \mathrm{kN} / \mathrm{m}^{2}$ from Eq. (3). Referring to Fig. 4, in which $s$ is plotted versus $q$ using Eq. (3) for different values of $\Gamma$ and assuming $a, t, h$ and $E$ as above, we see that crack length is very sensitive to $\Gamma$ for small values of $q$, but that the sensitivity diminishes as $q$ grows. From Fig. 3 however, we see that the 
adhesion is sensitive not only to the crack length but also to the beam deflection profile. We take advantage of this feature in our experiments by measuring point by point beam deflection profiles, so that even at high applied loads our sensitivity to adhesion is significantly better than Fig. 4 would imply. Nonetheless, the adhesion sensitivity depends on crack length as well as applied force. Assuming $10 \mathrm{~nm}$ out of plane resolution from the interferometry, at $q \sim 10 \mathrm{kN} / \mathrm{m}^{2}$ and $s=1665 \mu \mathrm{m}$, the sensitivity is approximately $0.03 \mu \mathrm{J} / \mathrm{m}^{2}$, while at $q \sim 70 \mathrm{kN} / \mathrm{m}^{2}$ and $\mathrm{s}=300 \mu \mathrm{m}$, the deflections are dominated by the applied force and the experimental sensitivity is about $10 \mu \mathrm{J} / \mathrm{m}^{2}$.

Eq. (3) applies for $G_{q} \geq 0$, or for

$$
q \leq q_{\max }=\frac{72 h D}{a^{3}(4 s-3 a)}
$$

At $q=q_{\max }, G_{q}=0$ and beam mechanics (ignoring adhesion) describe the deflections. In Fig. 3, $d^{2}\left(v_{2}(s)\right) / d x^{2}=0$ for $q=q_{\max }$. At the crack tip, the moment due to adhesive forces therefore vanishes, and a pure negative (upwards) shear force is imposed by the substrate. For $q>q_{\max }$ the deflections $v_{2 q}(x)$ penetrate the substrate and therefore no longer apply. If in fact the adhesion energy were negative (corresponding to repulsive, e.g., magnetic forces), an additional negative (counterclockwise) moment can be added to the constants of integration (Eq. A3) to ensure that that the deflections avoid dipping into the substrate.

With the JKR assumption of adhesion forces operating only over the contact zone, the beam theory equations reveal a point reaction and moment at $x=s$ which are imposed by the rigid substrate. Beyond the crack tip $(x>s)$, the beam deflection is constant at $v(x)=h$. and there is no loading over the attachment length $d$ for $x>s$. The reaction force applied by the substrate at the crack tip is equal and opposite to the shear force $V(s)$ and is given by

$$
V(s)=-D \frac{d^{3}}{d x^{3}}\left(v_{2}(s)\right)=-\frac{q a^{3}}{s^{2}}+\frac{\left(q a^{4} / 2+12 h D\right)}{s^{3}}
$$


In Fig. 5, we plot this relationship. Here a value for $\Gamma$ is held constant, a value for $q$ is assigned, $s$ is found from Eq. (3), and Eq. (5) is then applied to find the reaction force $P_{t i p}=w V(s)$ (calculated for the experimental beam width $w=10 \mu \mathrm{m}$ ). We see that for small values of $q$, the reaction force is tensile and increases with $\Gamma$ because there is more tension at the crack tip for larger adhesion. For large values of $q$, the force becomes compressive, because $q$ now dominates over adhesion. The shear force diagram in the vicinity of the crack tip for the case of large compression is shown in Fig. 6(a).

The loading situation as shown in Fig. 6(a), in which the compressive force is maximized very near the crack tip, is quite different from that in surface force apparatus adhesion hysteresis experiments on crossed cylinders, in which the compressive force is maximized at the center of the contact zone. We shall see that this compressive loading near the crack tip can allow us to determine if there is a pressure sensitive component to adhesion during crack healing in these experiments. Such a signature is not observed in spherical geometry adhesion hysteresis experiments.

Besides the JKR approximation, another well-known model for adhesion between contacting bodies is that of Derjaguin, Muller and Toporov [16], often referred to as the DMT model. In the DMT approximation, surface forces only outside of the areas of contact are considered in calculating adhesion, but these forces have no effect on the elastic deflections of the bodies. This approximation tends to be valid when the bodies are elastically hard [17]. For the necessarily compliant beams in this study, the DMT approximation is not physical. If we assume that surface forces act outside the contact zone, then only one shape is possible in order to balance forces. This shape can be solved for by iterative calculation, as described in the next section.

\section{2) Finite element modeling}

While the analytical model above provides helpful insight, it is inadequate for the quantitative reduction of our experimental data. There are several reasons for this. First, in Fig. 1, the distributed load applied by electrostatic forces is not uniform, because the gap between the beam and the substrate changes as a function of distance $x$ from the support post. The electrostatic force at each point along the actuation pad is 
$q(x)=\frac{\varepsilon_{o}}{2}\left(\frac{V_{p a d}}{h-v_{1}(x)}\right)^{2}$,

where $V_{p a d}$ is the applied voltage and $\varepsilon_{o}$ is the permittivity of air. In this more complicated situation, an iterative solution is required to solve for the local applied forces and deflections. The solutions were found using the ABAQUS [18] finite element method (FEM) code, with the electrostatic force law implemented by means of a subroutine. Note that in Figs. 4 and 5, the $x$ axis is plotted in two ways. The lower $x$-axis corresponds approximately to the applied voltage in our experiments below. The correspondence is not exact because the load is not uniform ( $q$ was calculated at $x=55 \mu \mathrm{m}$ ) and also because the adhesion affects the deflections, which in turn affect the force. The second reason FEM analysis is required to reduce the data is that nonidealities such as support post compliance and fringing fields at the edges of the beam could also be incorporated into the FEM modeling, as discussed further in the results section. The third reason is that FEM allows incorporation of surface forces near the crack tip, as discussed in the next paragraphs.

The adhesion was calculated by FEM in two ways. First, clamping the beam to the substrate at small increments.of $s$, we checked that the FEM modeling agreed with the analytical results. Second, a force law between two smooth surfaces which can approach to within an approach distance $D_{0}$ was assumed. While this is a reasonable approximation for our surfaces in most cases as shown below, the details of the effect of the roughness on local adhesion deserve further consideration beyond the scope of this work. Also, we shall not consider here the details of the stress field at the crack tip, such as the mode mixity and its relationship to interfacial slip, or its interplay with roughness. We implemented the surface force law in a general fashion, with integration of the force per unit area from $D_{\mathrm{o}}$ to infinity defining the surface energy. $D_{0}$ is established by the roughness of the surfaces as seen below. A simple linear force law over an effective range of $5 \mathrm{~nm}$ was used for most of the data reduction. Other more physical force laws such as a $(1 / \text { distance) })^{3}$ dependence (corresponding to van der Waals forces for two parallel plates) were also investigated but gave identical results as long as the active distance was approximately the same. The deflection of the beam was then calculated as a competition 
between elastic restoring forces in the beam, and surface forces at the crack tip. When coupled with the experimental data, the FEM allows us to accurately evaluate adhesion energies.

One effect of the surface forces at the crack tip is to cause a slight further flexure of the adhered beam compared to the JKR model. Thus, for a given surface force law, the FEM deflections are accurate within the limitations of the element size (we used 1-D beam elements every $2.5 \mu \mathrm{m}$, and checked for good convergence with 2-D mesh elements and more closely spaced elements down to $0.5 \mu \mathrm{m})$. In ref. [19], we shall detail the differences in the flexures when the surface force laws are incorporated. The main result is that independent of the surface force law, and independent of $q$, the deflections are very similar. That is, the JKR surface force law is an extremely good approximation to more physical surface force laws. This implies that the results we calculate in the results section below are sensitive only to the adhesion value, rather than to the particular surface force law we assume. A second effect is that implementing the surface force law more closely reflects the real tractions in the crack tip area than does the JKR assumption. As seen in the schematic Fig. 5(b), surface force tractions reduce the beam shear force to the left of the crack tip. Also, going beyond simple beam theory, a distributed pressure reaction force rather than a point reaction force exists beyond the crack tip in the attachment region $s \leq x \leq L$. There is a peak in the pressure near the crack tip. and for $x>L+t$, the reaction is equal to the pressure due to the surface forces. To solve accurately for the reactive pressure in the substrate requires a highly refined mesh in that regime.

\section{Experimental}

We fabricated polycrystalline silicon (polysilicon) cantilever beam test structures with an actuation pad near the support post. Their fabrication is described in detail in ref. [20], and a side view is shown schematically in Fig. 1. The thickness of the beams was $2.00 \mu \mathrm{m}$ and their height $h$ was $2.28 \mu \mathrm{m}$. The length of the actuation pad in Fig. 1 is $50 \mu \mathrm{m}$, beginning at $13 \mu \mathrm{m}$ and ending at $63 \mu \mathrm{m}$ from the support post. The rms roughness of the polysilicon is $2.5 \mathrm{~nm}$ with approximately a $0.5 \mu \mathrm{m}$ distance between local asperities, as measured by atomic force microscopy [21]. Before the cantilevers are rendered free standing, they are encased in a matrix of silica as a result of the fabrication process. The samples are then dipped in hydrofluoric acid, which removes the silica but does not perturb the polysilicon. After transferring from HF to 
water and through a series of miscible solvents, they were coated with perfluorodecyltrichlorosilane (FDTS, $\mathrm{C}_{10} \mathrm{H}_{4} \mathrm{~F}_{17} \mathrm{SiCl}_{3}$ ) in isooctane according to the procedure described in ref. [5]. FDTS is a silane coupling agent which nominally is deposited in monolayer form, with hydrophobic fluorinated tail groups remaining exposed [22]. However, in octadecyltrichlorosilane (ODTS, $\mathrm{C}_{18} \mathrm{H}_{37} \mathrm{SiCl}_{3}$ ), a better studied monolayer lubricant, questions remain about the nature of the bonding of the silicon atom to the substrate (covalent versus hydrogen bonded) [23]. Also, the degree of polymeric cross linking between siloxane bonds in the interface may be limited by the strain resulting from the steric constraint that the hydrocarbon chain van der Waals radius is greater than the siloxane bond length [24]. The cross linking in FDTS may be further limited, because the fluorocarbon tail diameter is larger $(\sim 0.64$ $\mathrm{nm})$ than the hydrocarbon tail diameter $(0.50 \mathrm{~nm})$.

After the FDTS treatment, the structures were transferred from the solvents in reverse order to water. Because the contact angle of the treated surface with water is greater than $90^{\circ}$ ( $115^{\circ}$ was measured on flat Si samples), capillary action does not pull the beams into contact with the substrate when removed from water. Using interferometry, we confirmed that the cantilevers were free standing after drying up to the maximum fabricated length of $2000 \mu \mathrm{m}$. This implies that the coverage of the FDTS film was very good. The freestanding cantilevers were curved up very slightly due to a residual stress gradient through the thickness of the film, with a radius of curvature of approximately $1.6 \mathrm{~m}$, as measured by interferometry. This corresponds to an out-of-plane displacement of $300 \mathrm{~nm}$ for a $1000 \mu \mathrm{m}$ long beam.

In order to measure adhesion hysteresis and its dependence on relative humidity (RH), an environmental microprobing station was constructed, schematically represented in Fig. 7. A Michelson interferometer is attached to a $5 \mathrm{X}$ microscope objective, and enclosed by a latex membrane in a humidity controlled environment. Magnification including the eyepiece is 50X, and resolution is approximately $3 \mu \mathrm{m}$ in the plane of the sample. The samples were illuminated by monochromatic green light at $547 \mathrm{~nm}$ as characterized by spectrum photometry. Tilt of the reference surface in the Michelson interferometer was arranged such that background fringes on the substrate were parallel to the long axis of the cantilever beams. "Then, fringes on the cantilever beams directly indicate out-of-plane displacements. Interferograms were recorded on a computer controlled charged coupled device (CCD) camera. 
Humidity was introduced by flowing $\mathrm{N}_{2}$ gas through a stainless steel water vessel heated to $50^{\circ} \mathrm{C}$. Relative humidity $(\mathrm{RH})$ was monitored using an in-situ humidity probe. Electrical current between the actuation pad and ground was monitored on a picoammeter. The current remained in the $\mathrm{pA}$ regime up to $80 \% \mathrm{RH}$. There was no evidence of electrostatic breakdown between the actuation pad and the beams in spite of the high electric field $(0.8 \mathrm{MV} / \mathrm{cm})$ in the gap, consistent with the observations in ref. [25]. Even at $160 \mathrm{~V}$ and $99 \%$ humidity, the current did not exceed $100 \mathrm{nA}$. The mechanism for the current flow is likely surface conduction between the actuation pad and the landing pad or the support posts.

On one sample, tests were conducted at low RH ( $<5 \%$, dry nitrogen only), and 25 parallel cantilevers were subjected to thousands of actuation cycles. No qualitative effect on the adhesion or adhesion hysteresis was observed. However, after increasing the $\mathrm{RH}$, an increase in adhesion was noted at $90 \% \mathrm{RH}$ after several hours exposure. Another sample was then inserted and this phenomenon was investigated more carefully. This sample was first tested in a low RH ambient, while being viewed in-situ under interferometric conditions. Initially and at timed intervals of 1/2, 4, 7 and 10 hours thereafter, $V_{\text {pad }}$ was stepped through values of $0,50100,120$, 140 , and $160 \mathrm{~V}$ for "crack healing" and subsequently through $140,120,100,50$ and $0 \mathrm{~V}$ for "crack propagation". During the voltage stepping, the time between images was approximately 30 seconds. At $V_{\mathrm{pad}}=160 \mathrm{~V}$, the distributed force is large and the crack lengths are about $300 \mu \mathrm{m}$ long. This sequence was repeated at increasing $\mathrm{RH}$ levels of $<5 \%, 30 \%, 60 \%, 80 \%, 90 \%$ and $95 \%$. At each value of $V_{\text {pad, }}$, an interferometric image was recorded and stored for future analysis. From the stored interferograms, linescans along individual beams were extracted using an image analyzer program [26]. These contain fringe information representative of out-of-plane $v(x)$ deflections, and were interpolated to nm-scale deflection data along the length of the beam by a computer program.

\section{$\underline{\text { Results }}$}

The initially suspended beams were brought into contact contact with the substrate by applying voltage to the actuation pad at low $\mathrm{RH}$, and were then adhered with crack lengths $s \sim 1400 \mu \mathrm{m}$ after the voltage was relaxed, corresponding to an adhesion of $2.4 \mu \mathrm{J} / \mathrm{m}^{2}$. The voltage step cycles described above were then begun. Comparing the measurement at $V_{\mathrm{pad}}=0 \mathrm{~V}$ before and after a given voltage stepping cycle, adhesion of the beams was not observed to 
change significantly from 0 to $80 \% \mathrm{RH}$. After four hours exposure at $90 \% \mathrm{RH}$, an adhesion increase to $\sim 4 \mu \mathrm{J} / \mathrm{m}^{2}$ was noted, and a much more significant increase to as large as $400 \mu \mathrm{J} / \mathrm{m}^{2}$ was observed after a seven hour exposure [21]. We shall focus the present adhesion hysteresis analysis on the cycle taken initially at low $\mathrm{RH}$, and on the cycle at $\mathrm{RH}=90 \%$ after the seven hour exposure. As discussed in ref. [21], beams coated with ODTS showed only a very small adhesion increase due to exposure to humidity levels up to $99 \%$.

There was no apparent difference in interferograms of the cantilevers at increasing and decreasing values of voltage at low $\mathrm{RH}$. This is a qualitative indication that at low RH, there is no adhesion hysteresis. However, even when there is no hysteresis, adhesion changes along the length of the beam should be evaluated to determine the degree of adhesion non-uniformity due either to surface roughness or FDTS coating non-uniformities. Interferograms of the cantilever beam array exposed for 7 hours at $90 \%$ RH' show significant adhesion hysteresis. In Fig. 8 , comparing fringe patterns at $100 \mathrm{~V}$, the fringes end closer to the support posts for the decreasing voltage than for the increasing voltage. The effect is much more pronounced at $50 \mathrm{~V}$ and $0 \mathrm{~V}$, qualitatively indicating strong hysteresis. The beams in Fig. 8 are configured in an array of increasing length from bottom to top. This is to aid in the assessment of the release process to create the free standing beams. Here, we measure adhesion only on only those beams which are attached over a long length $d$, per. ref. [14].

Other important experimental observations were: (1) The adhesion increase at high RH was qualitatively the same on the sample which was subjected to many contact cycles at low RH and the sample which was not. Also, cantilever beam arrays that had not been actuated at all were actuated once at high RH after exposure to high RH and showed an adhesion increase similar to those which had been actuated many times. These observations indicate that the adhesion increase is primarily related to exposure of the surface to high $\mathrm{RH}$, rather than to the cycling of the samples. (2) Note that in Fig. 8 the behavior of individual beams is quite different, especially as voltage is relaxed. We believe that this is a signature of the non-uniform nature of the adhesion increase mechanism, as described in the discussion section.

(3) Cantilever beam arrays which were exposed to high RH, but had not been actuated, were also tested at low RH. These displayed an adhesion very similar to beams which had not been exposed to high RH. This suggests that capillary action plays a strong role in the adhesion increase. 
To quantify the adhesion along the length of the beams accurately, we found that it was important to incorporate four non-idealities into the modeling, as will be detailed in ref. [Knapp, $2000 \# 245]$. Here, we briefly list the corrections in order of importance. First, the compliance of the support post was very important in obtaining measurements at high actuation pad voltages. It was determined directly by measuring the difference between deflections of $1000 \mu \mathrm{m}$ long cantilever beams (not yet touching the substrate) with increasing actuation voltages applied, and comparing to varying modeled support post compliances until good agreement was found. Second, the electrostatic fringing field at the outside edges of the beams was added in by use of a simple formula [28]. Third, a linear correction to the data was made to account for a small degree of angular offset between the background substrate fringe direction and the beam direction. Fourth, the initial curvature of the beams due to stress gradient (caused by nonuniform stress through the thickness of the film [27]) was measured and incorporated into the modeling. With accurate characterization of these non-idealities, adhesion of beams contacting the substrate at various loads applied was extracted by minimizing the difference between modeled and measured $v(x)$ values at a given applied voltage, as described next.

In Figs. 9(a) and (b) we show measured and modeled deflections of an individual beam from Fig. 8 at increasing and decreasing voltages respectively. The modeled deflections represent an optimum fit to the data in which the only free parameter is the adhesion. The best fit is determined by choosing the minimum in average root mean square error per pixel between measured and modeled deflections as shown in Fig. 10(a)-(c). In these plots, we utilize the full length of the measured deflection curve for error minimization. The minimum error is on the order of uncertainty from the interferometry measurements $(<10 \mathrm{~nm})$. This indicates that the modeling is good and that the important physical mechanisms have been incorporated and quantified well. In Fig. 10, the depth of the minimum successively decreases with increasing applied voltage, as is to be expected from the discussion on sensitivity versus applied load. We shall discuss the fitting procedure and results in more detail in ref. [19].

Using this methodology, we plot the results of the adhesion hysteresis measurements versus applied voltage in Fig. 11 for two beams at low RH and for four beams in Fig. 12 at high RH. (The beam numbers are counted from the top of the image). For the beams at low RH in Fig. 11, the adhesion is almost independent of applied voltage, indicating that the adhesion is uniform along the length of the beam, with values ranging from 0.5 to $3 \mu \mathrm{J} / \mathrm{m}^{2}$. In fact, adhesion 
was observed to be in the range of $1-10 \mu \mathrm{J} / \mathrm{m}^{2}$ and uniform along the beam length up to RH levels of $80 \%$, indicating that the FDTS film is uniformly deposited and relatively inert to water adsorption up to these levels. The beams in Fig. 12 at $90 \%$ RH were chosen to examine the range of adhesion increase during the actuation cycle, from 30 to $400 \mu \mathrm{J} / \mathrm{m}^{2}$. In Fig. 12 , we see that the adhesion begins to increase with increasing voltage above $100 \mathrm{~V}$. Although the uncertainty is relatively large for the values of voltage above $100 \mathrm{~V}\left(\sim 20 \mu \mathrm{J} / \mathrm{m}^{2}\right.$ at $\left.V_{\mathrm{app}}=160 \mathrm{~V}\right)$, the large difference between the low and high humidity cases of Figs. 11 and 12 indicates that the adhesion increase with increasing voltage is a real effect, and not an artifact of sensitivities in the adhesion extraction algorithm.

To begin to understand the physical mechanism for the adhesion increase, we compared the landing pad areas (see Fig. 1) of the samples which had been exposed to high RH to the landing pads of samples which had been kept in air (in RH generally $\leq 40 \%$ ) by atomic force microscopy (AFM). A Digital Instruments (Santa Barbara, CA) Nanoscope IILA was used with silicon nitride cantilevers. The AFM was conducted in air after the samples were removed from the high RH environment. Results are shown in Fig. 13, where a number of agglomeration sites (mounds) are seen for samples exposed to high RH (Fig. 13(b)). Such mounds were not observed in samples not exposed to high RH (Fig. 13(a)). The density of the mounds is approximately 0.1 to 0.2 per $\mu \mathrm{m}^{2}$, their heights are typically $10-25 \mathrm{~nm}$, but can be as large as 65 $\mathrm{nm}$, and the diameters are typically 500-1000 nm. A linescan of a relatively large mound is shown in Fig. 13(c). Smaller mounds may"be present but are difficult to distinguish from the 2.5 $\mathrm{nm}$ rms substrate roughness. The presence of these mounds was reported in ref. [21], and their origin is discussed further below.

\section{Discussion section}

No adhesion hysteresis is observed up to $80 \% \mathrm{RH}$.

It is clear from the experimental interferograms that there is minimal adhesion hysteresis for the FDTS-coated surfaces up to $80 \% \mathrm{RH}$. That is, these interferograms look identical at a given $V_{\text {pad }}$ independent of whether $V_{\text {pad }}$ is increasing or decreasing. Yet, hydrocarbon and fluorocarbon-tailed monolayer films deposited by the Langmuir-Blodgett technique exhibit adhesion hysteresis in surface force apparatus experiments [29,30], as well as in similar experiments between polydimethyl siloxane and fluoroalkylsiloxane coated mica [31]. The 
proposed mechanisms for the hysteresis are chain interdigitation when surfactant films are in an amorphous state [29], and molecular as well as submolecular changes in a fluorocarbon surfactant due to the bulky fluorocarbon groups [30]. Due to the atomically flat surfaces, the advancing contact (corresponding to crack healing) adhesion in those experiments is tens of $\mathrm{mJ} / \mathrm{m}^{2}$ (i.e., millijoules per square meter) while the receding contact (corresponding to crack propagation) adhesion can reach values several times larger.

In the present experiments, the surfaces exhibit a $\sigma=2.5 \cdot \mathrm{nm}$ rms roughness as measured by AFM (atomic force microscopy). This would seem to be a fairly smooth surface, and one might expect that there is still sufficient contact area that adhesion hysteresis would manifest itself. Indeed, as mentioned in the mechanics sections, our experimental resolution with respect to adhesion hysteresis at low voltages is such that a difference of $0.03 \mu \mathrm{J} / \mathrm{m}^{2}$ between healing and propagating crack energies should be detectable, well beyond the resolution for adhesion hysteresis in the surface forces apparatus.

There are two main mechanisms potentially contributing to adhesion between non-wetted rough surfaces. First, surfaces that are in intimate contact will increase adhesion because surface energy is reduced. Second, surfaces that are in close proximity will enhance adhesion due to van der Waals forces. We shall show next that this second component dominates for our surfaces. This will help to explain why there is no measurable adhesion hysteresis at low RH.

Although the roughness is quite small, the radius of curvature at the tip of individual asperities is $R \sim 100 \mathrm{~nm}$ as measured by AFM. This is a very small radius of curvature, and ignoring the effect of the very thin monolayer, the asperities are elastically very hard. In such a situation, the effect of adhesion on the real contact area $A_{R}$ is small. Therefore, we can consider the Greenwood-Williamson (GW) theory [32] to make initial estimates on the magnitude of $A_{R}$. In the GW theory, it is assumed that spherically capped asperities of radius $R_{P}$ are statistically distributed in height according to a Gaussian function with standard deviation $\sigma_{P}$. They contact a rigid smooth surface according to Hertzian mechanics. Normalizing for two rough surfaces as in the present situation, we take $R_{P}=50 \mathrm{~nm}$, and $\sigma_{P}=3.7 \mathrm{~nm}$. The order of magnitude calculations here do not depend strongly on the choice of these two values.

Before applying the GW calculations, we must first determine if the asperities are elastically or plastically compressed. According to GW, the plasticity parameter $\Psi$ is 


$$
\Psi=\left(\frac{E^{\prime}}{H}\right) \sqrt{\frac{\sigma_{P}}{R_{P}}},
$$

where $E^{\prime}=169 \mathrm{GPa}$ is the plane strain value of Young's modulus. of the rough surface material, and $H=13 \mathrm{GPa}$ is its hardness. If $\Psi<0.6$, the asperities are elastically compressed, and if $\Psi>0.9$, they are plastically compressed. Inserting these values into Eq. (7) results in $\Psi=3.5$. Therefore, the asperities are plastically compressed! This simplifies the calculation for $A_{R}$ such that.

$$
\frac{A_{R}}{A_{A}}=\frac{P_{A}}{H},
$$

where $A_{A}$ and $P_{A}$ are the apparent area and applied pressure respectively. Assuming a reasonable value of $P_{A}=220 \mathrm{~N} / \mathrm{m}^{2}$ (as will be justified later), we have $A_{R} / A_{A}=1.7 \times 10^{-8}$. (The value is slightly smaller if the elastic calculations are carried out). This is an extremely low ratio, and is due to the high value of $\sigma_{P} / R_{P}$ when compared with engineering surfaces. At this pressure, the adhesion due to areas of intimate contact $\Gamma_{A_{R}}$ is

$\Gamma_{A_{R}}=2 \gamma \frac{A_{R}}{A_{A}}$

With $2 \gamma \sim 10 \mathrm{~mJ} / \mathrm{m}^{2}$ the surface energy of the monolayer, we find $\Gamma_{A_{R}}=0.0005 \mu \mathrm{J} / \mathrm{m}^{2}$. This is significantly lower than the measured value of $\Gamma=2.5 \mu \mathrm{J} / \mathrm{m}^{2}$, and therefore the adhesion is indeed dominated by van der Waals forces of non-contacting areas, rather than by areas of intimate contact. This can be understood in terms of subsequent calculations which show that the contacting asperities on average are many tens of microns apart. Meanwhile the average surface separation $D_{o}$ due to the surface roughness is only about $20 \mathrm{~nm}$ as can be seen from the following calculation. Using the data $\Gamma=2.4 \mu \mathrm{J} / \mathrm{m}^{2}, D_{o}$ can be determined from. 


$$
\Gamma=\frac{A}{12 \pi D_{o}^{2}}
$$

where $A$ is the Hamaker constant. $A$ ranges from $0.5 \times 10^{-19}$ to $2 \times 10^{-19} \mathrm{~J}$ for a variety of surfaces [33]. Using $A=0.5 \times 10^{-19}$ for fluorocarbon material, we find $D_{o}=23 \mathrm{~nm}$. From the derivative of Eq. (7), an average pressure due to the van der Waals interaction in the adhered region is $q=220 \mathrm{~N} / \mathrm{m}^{2}$.

Because van der Waals forces exhibit no hysteresis, the lack of adhesion hysteresis in these experiments at low $\mathrm{RH}$ is now well understood. At $V_{\mathrm{pad}}=0 \mathrm{~V}, q=220 \mathrm{~N} / \mathrm{m}^{2}$ due the van der Waals forces, with $\Delta 2 \gamma-20 \mathrm{~mJ} / \mathrm{m}^{2}$ for a typical adhesion hysteresis value, we expect using Eq. (9) an adhesion hysteresis $\Delta \Gamma=0.001 \mu \mathrm{J} / \mathrm{m}^{2}$. As stated in the mechanics section, the experimental sensitivity at $V_{\mathrm{pad}}=0 \mathrm{~V}$ is $0.03 \mu \mathrm{J} / \mathrm{m}^{2}$. Therefore, the very small adhesion hysteresis of these surfaces is beyond the experimental detection capability. As $V_{\text {pad }}$ increases, a similar argument can be made. For example, at $V_{\text {pad }}=160 \mathrm{~V}$, and using Fig. 5, $P_{A} \sim P_{\text {tip }} /(w t / 2)=50,000 \mathrm{~N} / \mathrm{m}^{2}$. Therefore, $A_{R} / A_{A}=3.8 \times 10^{-6}$ and $\Delta \Gamma=0.04 \mu \mathrm{J} / \mathrm{m}^{2}$, while the experimental detection limit is $10 \mu \mathrm{J} / \mathrm{m}^{2}$. Although $D_{o}$ near the crack tip will decrease due to the large pressure, calculation shows that the commensurate adhesion increase remains below this limit. Therefore, adhesion hysteresis is negligible at low values of $\mathrm{RH}$.

In light of the plastic deformation experienced by the asperities, it seems surprising that the adhesion does not begin to increase, especially as RH increases to $80 \%$. One might think that the asperities would no longer be covered by the monolayer, and that water could nucleate locally in these areas, giving rise to an adhesion increase. Two considerations argue against this notion. First, in interfacial force microscope adhesion and scratch experiments on thiol monolayers on gold and silane coupling agent monolayers on $\mathrm{Si}$, no tendency for the films to be worn away was noted at GPa pressures [34]. Furthermore, even liquid layers between a small asperity tip and a substrate prove difficult to expel under high shear conditions. In such confined systems of just a few_layers, a crystalline state which is quite robust is thought to form [35]. Second, even if the monolayer is removed at the asperity tips at the $\sim 13 \mathrm{GPa}$ pressures 
encountered here, the area over which this occurs is so small that localized capillary action cannot have much affect on the adhesion.

\section{Large adhesion hysteresis is observed at high $\mathrm{RH}$}

At high RH values, two major effects were observed. First, the adhesion hysteresis is large, with $\Delta \Gamma$ from 30 to $400 \mu \mathrm{J} / \mathrm{m}^{2}$ as seen in Fig. 12. Second, the adhesion increases both as the crack heals and then repropagates. The agglomerated mounds observed in Fig. 13 suggest that these observations correlate strongly with a structural change in the film. As discussed in ref. [21] and described briefly here, we believe the mounds are actually isolated vesicles formed by a restructuring of the surface monolayer phase to a lyotropic bulk phase at high humidities. Lyótropic phases form between surfactant molecules and water, and exhibit viscoelastic properties. (See for example ref. [36]). Essentially, very soft and large radius of curvature asperities have developed on the surface as a result of this restructuring at high $\mathrm{RH}$. The vesicles in Fig. 13 are three dimensional, and their volume is approximately that of the original monolayer. Because this process occurs over the course of hours, we surmise that a diffusional process leading to a more stable bulk phase consisting of the vesicles has taken place. The vesicles are surrounded by lower lying areas which are likely hydrophilic because they are no longer covered by the coupling agent.

The vesicles themselves are likely hydrophobic, because if the adhesion increase at high $\mathrm{RH}$ were uniform along the length of the beams, the cracks would heal spontaneously. However, even at $90 \%$ and greater $\mathrm{RH}$, this was not observed - crack lengths decreased only as a result of a pad actuation cycle. This is in striking contrast to the case of hydrophilic beams, where we observed spontaneously decreasing crack lengths with increasing humidity over a range from 30 to $95 \% \mathrm{RH}$ [37]. In that case, nanometer size capillaries at the crack tip do work against the strain energy of the beam to heal the crack. Here, the protruding vesicles likely remain hydrophobic, while the areas which are uncovered become hydrophilic. We can think of the surface in Fig. 13 as hydrophobic islands rising above a hydrophilic sea. This leads to a nonuniform adhesion on the surface, with the scale of the non-uniformity being microns as defined by the distance between the vesicles. We now explain the main characteristics of the adhesion

- hysteresis curves for the four adhered beams at high RH shown in Fig. 12. 


\section{A) Crack healing (increasing voltage) at high $\mathrm{RH}$}

As $V_{\text {pad }}$ is increased from 0 to $50 \mathrm{~V}$ in Fig. 12, $\Gamma$ decreases from $\sim 3$ to $\sim 1 \mu \mathrm{J} / \mathrm{m}^{2}$. Analysis of the data indicates that the crack length remains very nearly the same from 0 to $50 \mathrm{~V}$, with the concomitant decrease in adhesion. The increase from 0 to $50 \mathrm{~V}$ provides only a small crack healing force. If adhesion were constant at $3 \mu \mathrm{J} / \mathrm{m}^{2}$, the crack would heal approximately $100 \mu \mathrm{m}$. This indicates that in the vicinity of the crack tip, surface forces are not locally available to do work on the beam. We can understand this in the following sense: if the protruding vesicles are hydrophobic, they will resist crack healing. As seen from Fig. 5, pressure which might compress the vesicles is very small (or tensile) at $50 \mathrm{~V}$, and therefore it is difficult for the cracks to heal beyond the first vesicle encountered.

When pad voltage is increased to $100 \mathrm{~V}$, sufficient driving force exists to force the cracks to heal some $200 \mu \mathrm{m}$, and the apparent $\Gamma$ sometimes increases and sometimes decreases relative to the values at 0 and $50 \mathrm{~V}$. If the crack tip reaches an area of locally high adhesion within the hydrophilic sea, surface forces will do more work on the beam to pull it further in, resulting in a larger value of adhesion. On the other hand, if it reaches a hydrophobic island it does not heal further, a lower value of adhesion is inferred. Note from Fig. 5 that the compressive force applied near the crack tip remains small at $V_{\text {pad }}=100 \mathrm{~V}$.

$\Gamma$ increases monotonically as applied voltage is increased from $120 \mathrm{~V}$ up to $160 \mathrm{~V}$. At higher voltages, compression near the crack tip may act to deform the compressible viscoelastic vesicles seen in Fig. 13(b). This would decrease the separation between the surfaces in the vicinity of the crack tip, allowing lower lying hydrophilic areas to come into contact, and causing the monotonically increasing adhesion with increasing voltage.

We can test this idea quantitatively. Recalling the discussion for Fig. 5. the magnitude of force at the crack tip is on the order of hundreds of nanonewtons at the highest voltage. Near the crack tip, this load will be borne by an individual vesicle because as seen in Fig. 13 they are spaced microns apart. From simple Hertzian mechanics, the deformation of the vesicles will be

$$
\Delta=\left(\frac{P_{t i p}^{2}}{K^{2} R}\right)^{1 / 3}, \quad-
$$


where $P_{t i p}$ is the compressive load applied by the beam to the vesicle, $K=(4 / 3)\left(E /\left(1-v^{2}\right)\right)$ is proportional to the modulus of the vesicle, and $R$ is the radius of curvature of the vesicle. At $V_{\mathrm{pad}}=160 \mathrm{~V}$, the value of $q$ from Eq. (5) will be approximately $70 \mathrm{kN} / \mathrm{m}^{2}$, resulting in $V(s)=-550$ nN per Fig. 5. The radius of curvature of the polymeric vesicles is $a_{o}^{2} / 2 \delta$, where $a_{o}$ is the vesicle radius and $\delta$ is the vesicle height. From the results section, we have $a_{o}=0.2 \mu \mathrm{m}$ and $\delta=20 \mathrm{~nm}$, resulting in $R=1 \mu \mathrm{m}$. Estimating $K=1 \mathrm{GPa}$ for the polymeric vesicles (typical polymers have modulus values of 1-3 GPa, but we expect a compliant structure here), and inserting these values into Eq. (8) results in $\Delta=6.7 \mathrm{~nm}$. Furthermore, the contact area is $\pi R \delta$, and the average pressure will be $25 \mathrm{MPa}$. This is beyond the hardness of many polymeric materials. Because of this high pressure and because the vesicles are likely viscoelastic, subsequent deformation beyond $\Delta=6.7 \mathrm{~nm}$ is to be expected. This is evidence that the adhesion is pressure sensitive when the monolayer reconfigures into isolated vessicles.

The above calculation supports the notion that the adhesion increase during crack healing is in fact due to increasing contact area under the applied load near the crack tip. Once the hydrophobic vesicles are sufficiently deformed, locally hydrophilic areas can bridge across the surfaces as seen in Fig. 14, which will subsequently significantly increase the measured adhesion. The monotonic increase in adhesion with voltage from 120 to $160 \mathrm{~V}$ indicates that more sites come into contact as the load is increased. The result is strikingly different from adhesion hysteresis experiments where spheres come into contact, and exhibit adhesion increase only upon crack repropagation [29-31]. The reason is that in the case of two spheres coming into contact, the pressure maximum is at the center of the contact area and far away from the crack tip, while in the present case the maximum is very near the crack tip. The emergence of the low modulus hydrophobic islands in the hydrophilic sea at the high RH levels, and their subsequent deformation due to the compression very near the crack tip, is the apparent mechanism for the increase in adhesion during crack healing in the present work.

After prolonged exposures of 40 hours at $\mathrm{RH}=95 \%$, adhesion was observed to continue to increase up to 500 to $1000 \mu \mathrm{J} / \mathrm{m}^{2}$ [21]. Hydrophilic surfaces approach $140 \mathrm{~mJ} / \mathrm{m}^{2}$ at these humidity levels [36], because the surface can be fully wetted. This maximum value for the hydrophobic surface can be understood in terms of Fig. 5. There we see that for large values of $\Gamma$, compressive force can no longer be generated in the vicinity of the crack tip at the maximum 
value of $V_{\text {pad. }}$ The pressure reaches 0 at $V_{\text {pad }}=160 \mathrm{~V}$ at $\Gamma=500 \mu \mathrm{J} / \mathrm{m}^{2}$, but small increases in adhesion beyond $500 \mu \mathrm{J} / \mathrm{m}^{2}$ can be expected if there are locally high areas of adhesion. Consequently, adhesion apparently cannot increase significantly beyond the $\mathrm{mJ} / \mathrm{m}^{2}$ range for the restructured surfaces in these experiments.

\section{Crack propagation (decreasing voltage) at high $\mathrm{RH}$}

In Fig. 8 we see that the adhesion is non-uniform from one beam to the next at the end of the actuation cycle. We attribute this to a statistical distribution of the vesicles. If there is a locale in which locally fewer vesicles and more hydrophilic surface area is present, this will give rise to greater adhesion. This will be observed not only from beam to beam, but within a beam as well, and helps to explain the characteristics during c̈rack propagation.

As $V_{\text {pad }}$ is lowered from $160 \mathrm{~V}$, the adhesion initially decreases or remains constant as seen in Fig. 12. Analysis of the deflection curves shows that the crack propagates in this region, indicating there is sufficient restoring force in the highly deformed beam to move past regions of high adhesion. However, as voltage decreases to $100 \mathrm{~V}$ or less, examination of the FEM results indicates that crack propagation ceases. The crack tip, which samples the adhesion continuously along the length of the crack during crack propagation, stops propagating at a site of high adhesion energy. The pinning of the crack gives rise to an apparent increasing adhesion as the

applied voltage continues to decrease. This explains why the energies appear to increase as $V_{\text {pad }}$ is lowered below $100 \mathrm{~V}$. Also, at $V_{\text {pad }}=0 \mathrm{~V}$, the apparent adhesion value is a lower bound. The exception is for beam 10 (Fig. 12c), which did not propagate at all as $V_{\text {pad }}$ was lowered from $160 \mathrm{~V}$. This beam exhibited the highest adhesion of the four beams in Fig. 12. For this beam, the crack tip probed a site of high local adhesion at $V_{\text {pad }}=160 \mathrm{~V}$.

The observation that beams exposed to high $\mathrm{RH}$, and tested at low $\mathrm{RH}$. showed no adhesion increase is consistent with the picture of the hydrophilic sea. Namely. at low RH, the hydrophilic areas will no longer be wetted, and therefore will not act to increase the adhesion.

\section{Summary and Conclusions}

We have developed the mechanics to investigte adhesion hysteresis of cantilever beams adhered to a substrate, both by an analytical approach as well as by finite element modeling (FEM). A key result of the mechanics is that for sufficiently high applied loads. a compressive load must be supported near the crack tip where the beam contacts the substrate. This is 
different from SFA adhesion hysteresis experiments in which the pressure is maximized away from the crack tip. From this we have some reason to suspect a different adhesion hysteresis characteristic than has previously been measured if the material near the crack tip is compressible.

A silane coupling agent (FDTS) monolayer was applied to the silicon microcantilevers in order to minimize susceptibility of adhesion to various $\mathrm{RH}$ ambients. To determine peformance limits of the monolayer, multiple contact (up to tens of thousands of cycles) and increasing humidity experiments were conducted. The RH experiments showed a significant adhesion increase, and this effect was studied in detail. Adhesion was tested at RH levels increasing from 0 to $95 \%$, with adhesion hysteresis test cycles conducted periodically up to 24 hours at a given RH level. FEM was required to quantify the adhesion at high loads because of the gapdependent load calculation. Furthermore, FEM allowed us to take into account such nonidealities as the support post compliance and the fringing fields from the electrostatic loading. Also, we were able to incorporate more physical surface force laws than can be achieved with the JKR assumption.

At RH values up to $80 \%$, negligible adhesion increase with applied load. as well as negligible adhesion hysteresis was observed. This is explained by the average separation between the surface being large enough that reversible van der Waals forces dominate the adhesion. Adhesion values did not increase significantly with voltage because the contribution to adhesion of increasing real contact area is very small, and because the reduction in average surface separation is small.

A substantial change in the adhesion and adhesion hysteresis was noted after a seven hour exposure at $90 \% \mathrm{RH}$. The adhesion increase is observed only upon actuation of the beams, rather than by spontaneous crack healing. Atomic force microscopy indicated that the silane coupling agent surface phase reconfigured into a bulk phase consisting of $\sim 20 \mathrm{~nm}$ high polymeric mounds, which we interpret to be vesicles. Formation of the vesicles leaves behind locally hydrophilic areas, but the vesicles themselves probably are hydrophobic and protrude above the surface, leading to a picture in which hydrophobic islands are surrounded by a hydrophilic sea. FEM quantification revealed that an adhesion increase is observed upon crack healing at high applied loads, in contrast to SFA adhesion hysteresis measurements. By calculating the applied load near the crack tip, we showed using Hertzian mechanics that the 
polymeric vesicles deform significantly, allowing lower lying hydrophilic areas to come into contact. When this occurs, capillaries can bridge the hydrophilic areas of the two surfaces, and the adhesion increases. This indicates that the restructured surface displays a pressure sensitive adhesion characteristic.

We have demonstrated that adhesion hysteresis of cantilever beams is a powerful tool for understanding aspects of reliability in surface micromachining. These experiments have enabled us to connect mechanics and chemistry at the microscopic level, and indicate that if wetting of MEMS surfaces can be prevented, adhesion and adhesion hysteresis can remain reasonably low.

\section{Acknowledgements}

Sandia is a multiprogram laboratory operated by Sandia Corporation, a Lockheed Martin Company, for the United States Department of Energy under Contract DE-AC04-94AL85000. We thank Robert Carpick for taking the AFM images. RM gratefully acknowledges additional support from Sandia National Laboratories, the National Science Foundation, and the Arnold and Mabel Beckman Foundation. We thank E. D. Reedy and J. E. Houston for carefully reading the manuscript.

\section{References}

[1] M. Madou, Fundamentals of Microfabrication (CRC Press, Boca Raton, Florida, 1997).

[2] R. Maboudian, Surface Science Reports 3,0209 (1998).

[3] T. I. Kamins, Polycrystalline silicon for integrated circuit applications (Kluwer, Boston, 1988).

[4] E. M. Russick, C. L. J. Adkins and C. W. Dyck, in Supercritical Fluids, Extraction and Pollution Prevention; Vol. 670, edited by M. A. Abraham and A. K. Sunol (American Chemical Society, Washington, DC, 1997), pp. 255-269.

[5] U. Srinivasan, M. R. Houston, R. T. Howe and R.:Maboudian, J. Micromech. Sys. 7 (2) 252 (1998).

[6] S. A. Henck, Tribol. Lett. 3239 (1997).

[7] M. E. McGovern, K. M. R. Kallury and M. Thompson, Langmuir 103607 (1994).

[8] J. B. Brzoska, I. B. Azouz and F. Rondelez, Langmuir 104376 (1994). 
[9] C. Carraro, O. W. Yauw, M. M. Sung and R. Maboudian, J. Phys. Chem. B 102 (23) 4441 (1998).

[10] J. N. Israelachvili, P. M. McGuiggan and A. M. Homola, Science 240189 (1988).

[11] J. N. Israelachvili, Y. L. Chen and H. Yoshizawa, J. Adh. Sc. Tech. 8 (11) 1231 (1994).

[12] T. Baumberger, Solild State Commun. 102 (2-3) 175 (1997).

[13] K. L. Johnson, K. Kendall and A. D. Roberts, Proc. Roy. Soc. Lond. A. $324-301$ (1971).

[14] M. P. de Boer and T. A. Michalske, J. Appl. Phys. 86 (2) 817 (1999).

[15] W. N. Sharpe, R. Vaidyanathan, B. Yuan, G. Bao and R. L. Edwards, J. Vac. Sci. Tech. 15 (5) 1599 (1997).

[16] B. V. Derjaguin, V. M. Muller and Y. P. Toporov, J. Colloid Interface Sci. 53 (2) 314 (1975).

[17] R. G. Horn, J. N. Israelachvili and F. Pribac, J. Colloid Interface Sci. 115 (2) 480 (1987).

[18] Hibbit, Karlsson and Sorensen Inc., (Pawtucket, RI, USA, ).

[19] J. A. Knapp and M. P. de Boer, to be submitted (2000).

[20] M. R. Houston, R. T. Howe and R. Maboudian. J. Appl. Phys. 81 (8) 3474 (1997).

[21] M. P. de Boer, T. M. Mayer, T. A. Michalske, U. Srinivasan and R. Maboudian, Langmuir submitted (1999).

[22] R. Banga, J. Yarwood, A. M. Morgan, B. Evans and J. Kells, Thin Solid Films 284-285 261 (1996).

[23] C. P. Tripp and M. L. Hair, Langmuir 111215 (1995).

[24] M. J. Wirth, R. W. P. Fairbank and H. O. Fatunmbi, Science 27544 (1997).

[25] J. Wibbeler, G. Pfeifer and M. Hietschold, Sensors and Actuators A A71 74 (1998).

[26] Analysis performed on using the public domain NIH image program; (National Institute of Health, ).

[27] D. Maier-Schneider, A. Koepurueluelue, S. Ballhausen Holm and E. Obermeier, J. Micromech. Microeng. 6436 (1996). .

[28] R. K. Gupta, 1997, Ph. D. Thesis, Ph.D. Thesis, MTT, Electrostatic pull-in test structure design for in-situ mechanical property measurement of MEMS, pages.

[29] Y. L. Chen and J. N. Israelachvili, J. Phys. Chem. 967752 (1992).

[30] S. Yamada and J. N. Israelachvili, J. Phys. Chem. B 102234 (1998).

[31] M. K. Chaudhury and M. J. Owen, Langmuir 929 (1993). 
de Boer et al., to be submitted to Acta Mater., Feb. '00

[32] J. A. Greenwood and J. B. P. Williamson, Proc. Roy. Soc. Lond. A. 295300 (1966).

[33] J. Israelachvili, Intermolecular and Surface Forces (Ácademic Press, New York, 1992).

[34] J. D. Kiely, J. E. Houston, J. A. Mulder, R. P. Hsung and X. Y. Zhu, Tribol. Lett. 7 (2-3) 103 (1999).

[35] S. Granick, Science 253 (5026) 1374 (1991).

[36] G. J. T. Tiddy, Physics Reports 57 (1) 1 (1980).

[37] M. P. de Boer, P. J. Clews, B. K. Smith and T. A. Michalske, Mater. Res. Soc. Proc. 518 131 (1998). 


\section{Appendix}

(1) Expression for $G$ in the presence of an externally applied distributed load $q$

Consider Fig. 1 in the main body of the paper. A cantilever beam of length $L$., thickness $t$ and height $h$ is adhered to the substrate over a distance $d=L-s$. If now a distributed load $q$ is applied over the interval $0 \leq x \leq a$, the crack length $s$ will decrease (if the adhesion is uniform along the length of the surface). In the absence of adhesion, simple beam mechanics can be used to determine where the beam will contact the substrate. However, because of the adhesion forces in the vicinity of the crack tip, the actual crack length $s$ will be somewhat shorter. Knowing the value of $s$, we can determine the adhesion according to the following beam mechanics. We use the JKR assumption in these calculations.

Over the interval $0 \leq x \leq a$, the equation for the deflections $v_{1 q}(x)$ is

$\frac{d^{4}}{d x^{4}}\left[v_{1 q}(x)\right]=\frac{q}{D}$

where $D=E I / w=E t^{3} / 12$ ( $E$ is Young's Modulus, $w$ is the width of the beam and $I=w t^{3} / 12$ is the moment of inertia of the beam). Integrating four times, we have

$v_{1 q}(x)=\frac{1}{D}\left(\frac{1}{24} q x^{4}+\frac{1}{6} C_{1 q} x^{3}+\frac{1}{2} C_{2 q} x^{2}+C_{3 q} x+C_{+q}\right)$

Over the interval $a \leq x \leq L$, the equation for the deflections $\nu_{2 q}(x)$ will be

$\frac{d^{4}}{d x^{4}}\left[v_{2 q}(x)\right]=0$

Integrating again four times,

$v_{2 q}(x)=\frac{1}{D}\left(\frac{1}{6}\left(q a+C_{l q}\right) x^{3}+\frac{1}{2} C_{5 q} x^{2}+C_{6 q} x+C_{7 q}\right)$ 
Here we have used $q a+C_{1 q}$ as the constant of integration in (A2) because the shear force in the beam at $x=a$ must be greater by $q a$ than at $x=0$.

For rigid support post and substrate, the boundary conditions are:

$v_{1 q}(0)=0, \frac{d}{d x}\left[v_{1 q}(0)\right]=0, v_{2 q}(s)=h$, and $\frac{d}{d x}\left[v_{2 q}(s)\right]=0$.

Continuity at $a$ requires that

$v_{1 q}(a)=v_{2 q}(a), \frac{d}{d x}\left[v_{1 q}(a)\right]=\frac{d}{d x}\left[v_{2 q}(a)\right]$, and $\frac{d^{2}}{d x^{2}}\left[v_{1 q}(a)\right]=\frac{d^{2}}{d x^{2}}\left[v_{2 q}(a)\right]$.

where the final boundary condition is because the moment cannot change instantaneously. Solving for the constants of integration in terms of known quantities gives

$$
\begin{aligned}
& C_{1 q}=-q a+q a^{3} / s^{2}-\left(q a^{4} / 2+12 h D\right) / s^{3}, \\
& C_{2 q}=q a^{2} / 2-\left(2 q a^{3}\right) /(3 s)+\left(q a^{4} / 4+6 h D\right) / s^{2}, \\
& C_{3 q}=C_{4 q}=0, \\
& C_{5 q}=\left(-8 q a^{3} s+3 q a^{4}+72 h D\right) /\left(12 s^{2}\right), \\
& C_{6 q}=q a^{3} / 6, \quad \text { and } \quad C_{7 q}=-q a^{4} / 24 .
\end{aligned}
$$

Inserting these values into (A1b) and (A2b), choosing $a=60 \mu \mathrm{m}, t=h=2 \mu \mathrm{m}, \mathrm{s}=1000 \mu \mathrm{m}, \mathrm{E}=160$ $\mathrm{GPa}$, and various values of $q$, we see the deflections in Fig. 2 in the main body. As expected, Eqs. (A1) and (A2) agree with Eq. (1) from the main body for $q=0$. 
In the absence of external force, the adhesion $\Gamma$ is as according to Eq. (2). To find the energy release rate $G_{q}$ in the presence of the external force, we must recognize that there are contributions both from work performed by external forces $F$, as well as from elastic energy $U_{E}$. Therefore,

$G_{q}=\frac{d F}{d s}-\frac{d U_{E}}{d s}$

The incremental work performed per unit width of the beam is

$$
\Delta F=q a \overline{\Delta v_{1}}
$$

where $\overline{\Delta v_{1}}$ is the average differential displacement over the interval $0 \leq x \leq a$, is defined by

$$
\overline{\Delta v_{1}}=\frac{1}{a} \int_{0}^{a}\left[v_{1}(x, s+\Delta s)-v_{1}(x, s)\right] d x
$$

In the limit where $\Delta s \rightarrow 0$,

$$
\frac{d F}{d s}=\frac{q^{2} a^{6}}{144 D s^{4}}(4 s-3 a)^{2}-\frac{q a^{3} h}{2 s^{4}}(4 s-3 a)
$$

The strain energy per unit width of the beam is

$$
U_{E}=U_{E 1}+U_{E 2}=\frac{1}{2 D}\left[\int_{0}^{a}\left(M_{1}(x)\right)^{2} d x+\int_{a}^{L}\left(M_{2}(x)\right)^{2} d x\right],
$$

where $M_{n}(x)=-D \frac{d^{2}}{d x^{2}}\left[v_{n}(x)\right]$. Solution to (A9) yields 
$U_{E}=\frac{-80 a^{6} s^{2} q^{2}+60 a^{7} s q^{2}-15 q^{2} a^{8}+8640 h^{2} D^{2}+36 q^{2} s^{3} a^{5}}{1440 s^{3} D}$.

The strain energy release rate term reduces to

$-\frac{d}{d s} U_{E}=\frac{18 D h^{2}}{s^{4}}-\frac{a^{6} q^{2}(4 s-3 a)^{2}}{288 s^{4} D}=G_{o}-\frac{a^{6} q^{2}(4 s-3 a)^{2}}{288 s^{4} D}$,

where $G_{o}$ is as defined by Eq. (2). Substitution of (A11) and (A8) into (A5) gives

$G_{q}=G_{o}+\frac{a^{6} q^{2}(4 s-3 a)^{2}}{288 s^{4} D}-\frac{q a^{3} h}{2 s^{4}}(4 s-3 a)$

For the present example, $G_{o}=7.68 \mu \mathrm{J} / \mathrm{m}^{2}$, while $G_{q}=1.67 \mu \mathrm{J} / \mathrm{m}^{2}$ with $q=10 \mathrm{kN} / \mathrm{m}^{2}$.

The solution (A12) applies for $G_{q} \geq 0$, or for

$q \leq q_{\max }=\frac{72 h D}{a^{3}(4 s-3 a)}$.

Eq. (A12) is plotted in Fig. Al for increasing values of $q$. We see that the crack healing term $d F / d s$ is negative. Meanwhile the strain energy release rate term $-d U_{E} / d s$ is positive and equal to $G_{o}$ for $q=0$, and diminishes with increasing $q$. As $q$ approaches $q_{\max }$, each term approaches zero. 


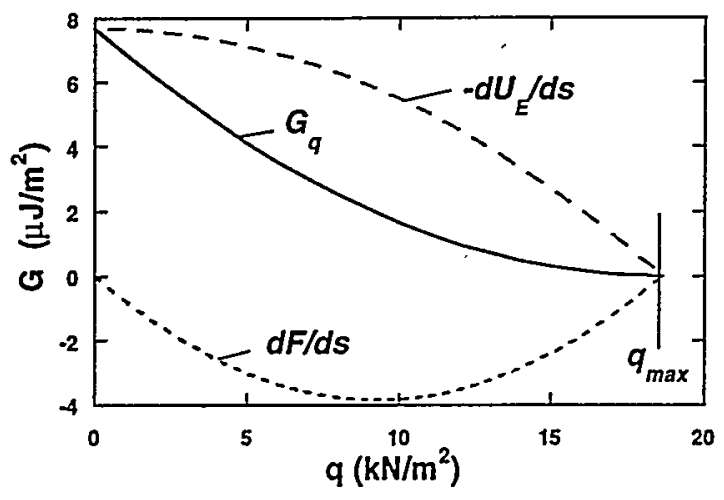

Fig. A1, $G_{q}=\frac{d F}{d s}-\frac{d U_{E}}{d s}$ vs $q$ for $s=1000 \mu \mathrm{m}$, and $t=h=2 \mu \mathrm{m}$. 


\section{Figure Captions}

Fig. 1 A cantilever beam subject to combined interfacial and electrostatic forces.

Fig. 2 Adhered cantilever beam with uniform distributed load $q$ applied for development of the analytical model.

Fig. 3 . Deflections for increasing values of $q$ and a constant value of $s=1000 \mu \mathrm{m}$.

Fig. 4 Dependence of crack length $s$ on adhesion $\Gamma$ in the presence of an externally applied load q. (JKR model, rigid support post, constant distributed pressure q).

Fig. 5 Reaction force $P_{\text {tip }}$ near the crack tip for a $10 \mu \mathrm{m}$ wide beam.

Fig. 6 Shear force diagram near the crack tip with (a) JKR or (b) more physical surface force law model.

Fig. 7 Environmental microprober

Fig. 8 Interferograms of adhesion hysteresis observed at RH=90\% after 7 hour exposure. The images are compressed by a factor of 3 in the horizontal relative to the vertical. The beams are $10 \mu \mathrm{m}$ wide. and spaced $5 \mu \mathrm{m}$ apart.

Fig. 9 Measured vs. modeled deflection data during (a) the "crack healing" process. and (b) the "crack propagation" process.

Fig. 10 Average rms per pixel curves versus adhesion at applied voltages of (a) $0 \mathrm{~V}$. (b) $100 \mathrm{~V}$ and (c) $160 \mathrm{~V}$. The adhesion is taken as the minimum in each curve.

Fig. 11 Adhesion vs. voltage for two beams at $\mathrm{RH}=0$.

Fig. 12 Adhesion vs. voltage for four beams at $\mathrm{RH}=90 \%, 7$ hour exposure.

Fig. 13 (a) Landing pad roughness at low RH. (b) agglomerated mounds of FDTS after exposure to high RH and (c) linescan across a large mound.

Fig. 14 Soft protruding areas compressed at the crack tip under large externally applied loads allow nearby hydrophilic areas to come into contact. 


\section{Figures}

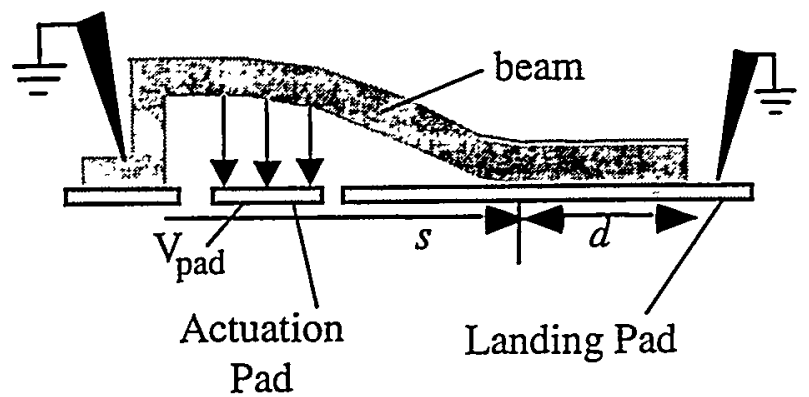

Fig. 1 A cantilever beam subject to combined interfacial and electrostatic forces.

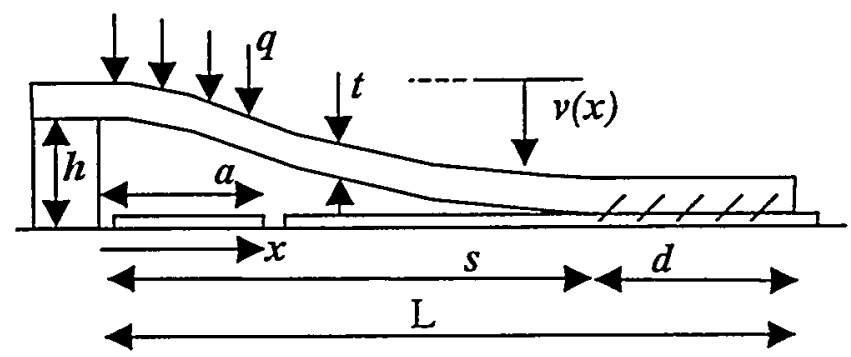

Fig. 2 Adhered cantilever beam with uniform distributed load $q$ applied for development of the analytical model.

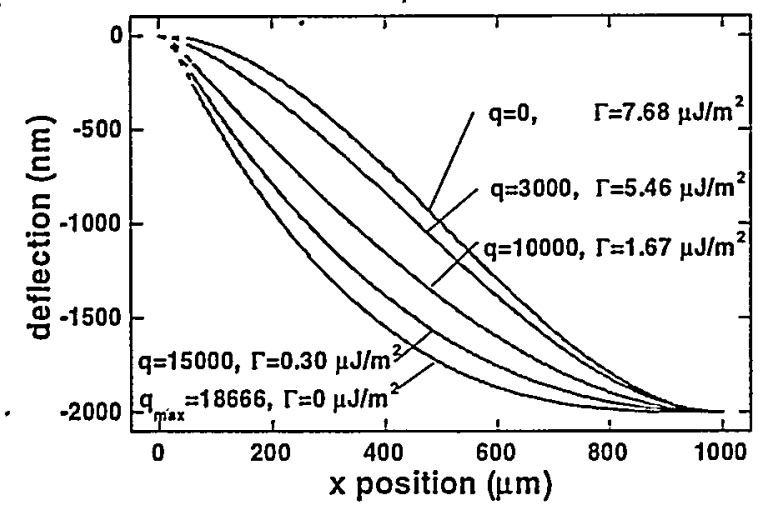

Fig. 3 Deflections for increasing values of $q$ and a constant value of $s=1000 \mu \mathrm{m} .\left(q-\mathrm{N} / \mathrm{m}^{2}\right)$ 


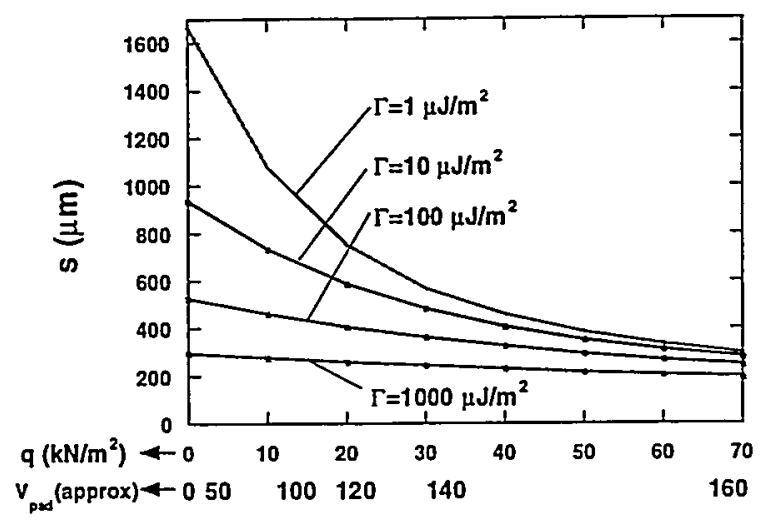

Fig. 4 Dependence of crack length $s$ on adhesion $\Gamma$ in the presence of an externally applied distributed load $q$.

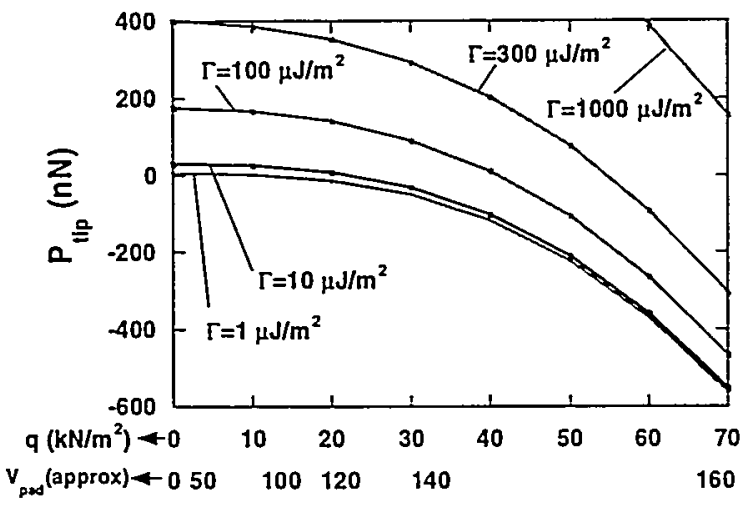

Fig. 5 Reaction force $P_{t i p}$ near the crack tip for a $10 \mu \tilde{m}$ wide beam

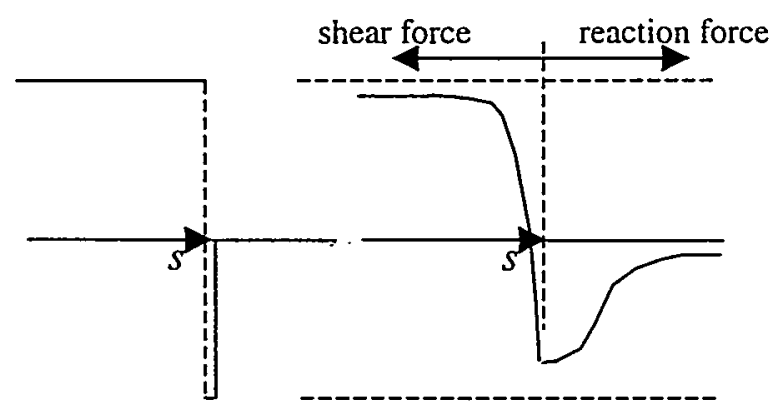

(a)

(b)

Fig. 6(a) Shear force loading diagram under (a) JKR assumption, (b) FEM solution with surface force law at the crack tip. 


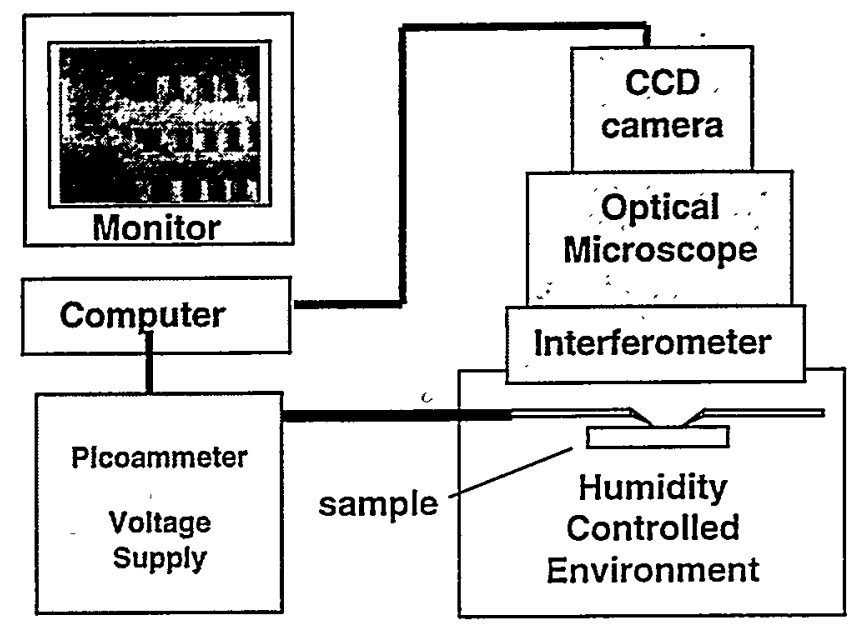

Fig. 7 Environmental microprober

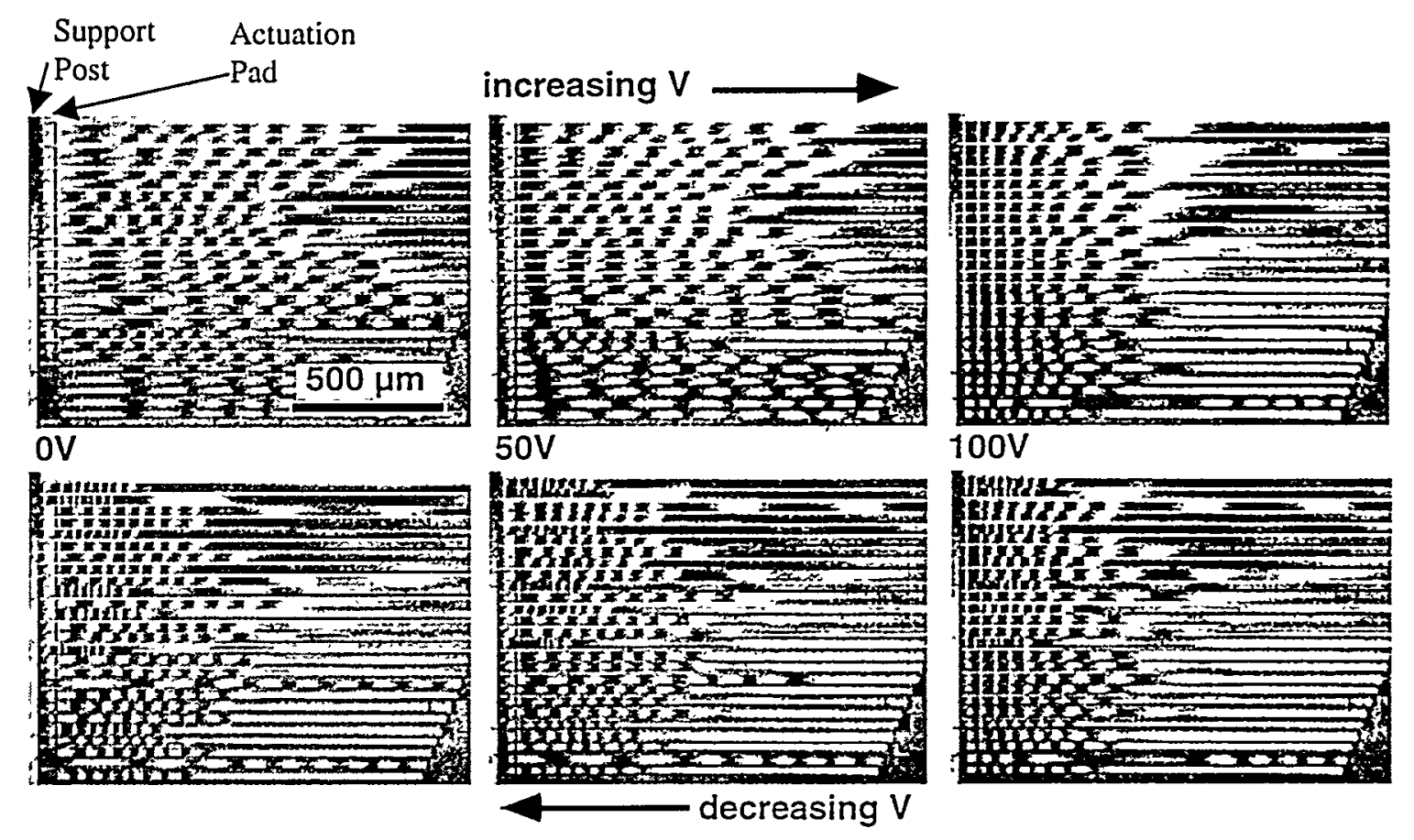

Fig. 8 Interferograms of adhesion hysteresis observed at $\mathrm{RH}=90 \%$ after 7 hour exposure. The images are compressed by a factor of 3 in the horizontal direction relative to the vertical. The beams run horizontal, are $10 \mu \mathrm{m}$ wide. and spaced $5 \mu \mathrm{m}$ apart. Their lengths are from $1500 \mu \mathrm{m}$ (bottom of each image) to $2000 \mu \mathrm{m}$ (top). Beams longer than $1700 \mu \mathrm{m}$ are cut off because of the CCD camera field limit. The voltage was increased up to 160 $\mathrm{V}$ before decreasing. 


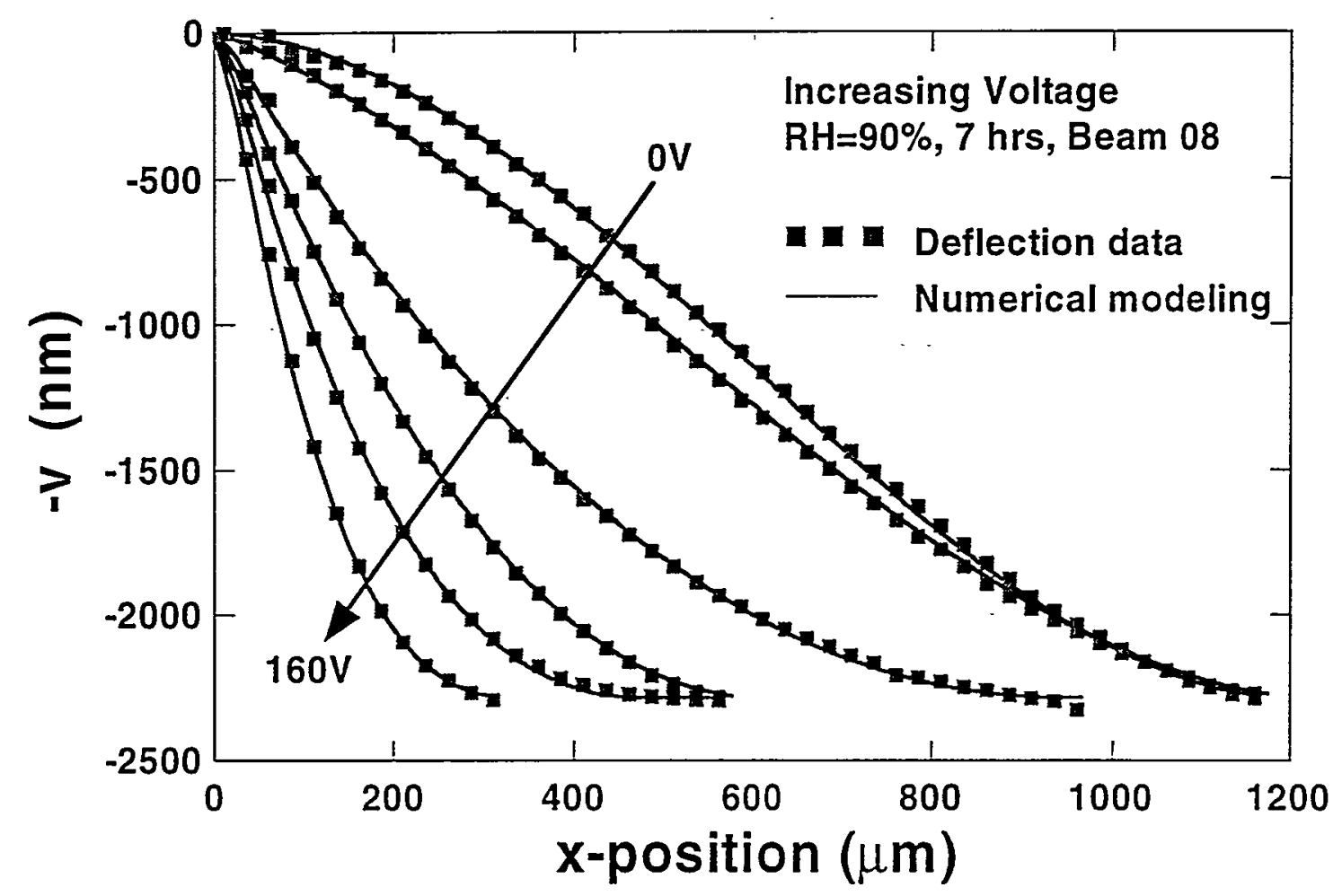

Fig. 9(a) Measured vs. modeled deflection data during the "crack healing" process.

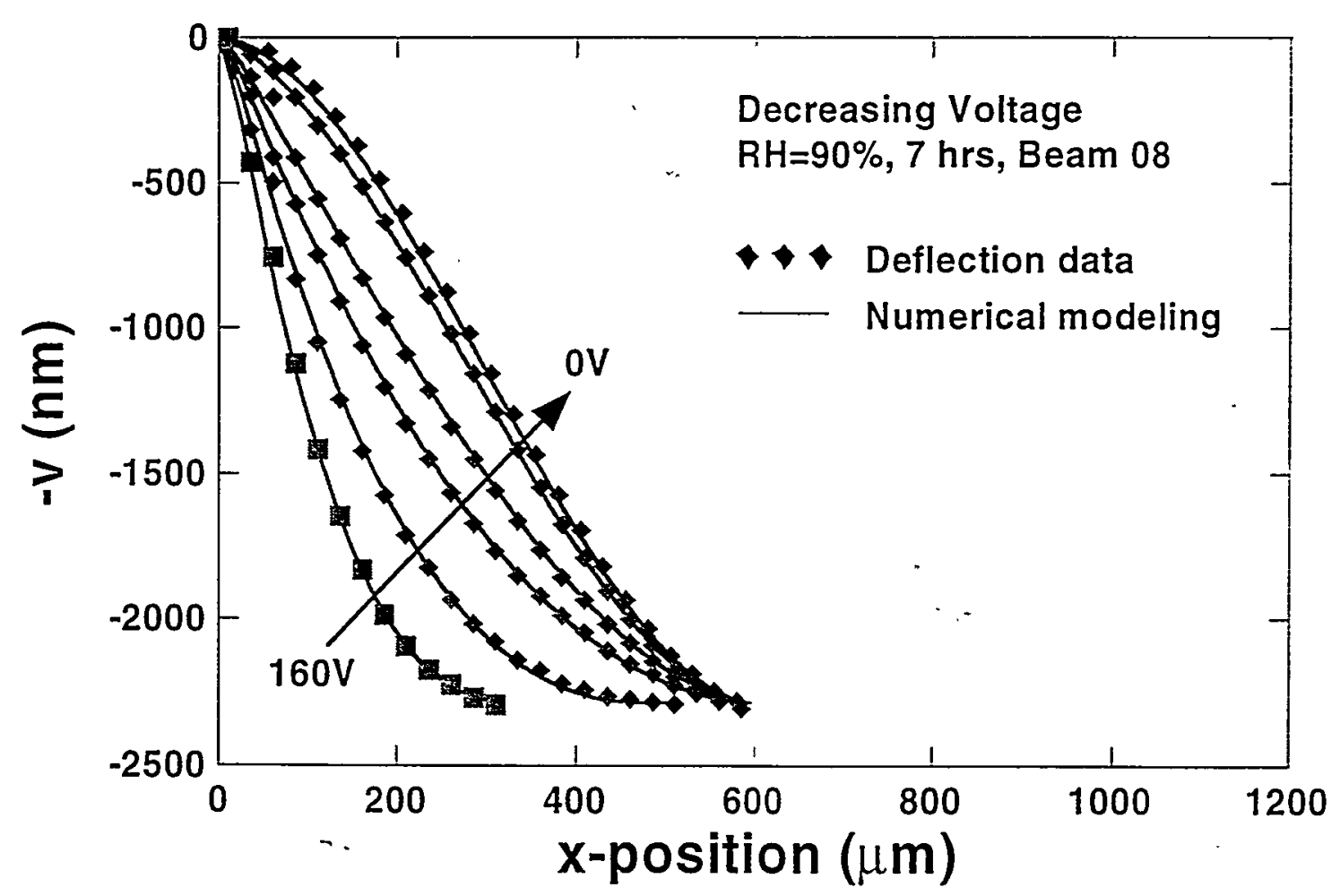

Fig. 9(b) Measured vs. modeled deflection data-during "crack propagation" 

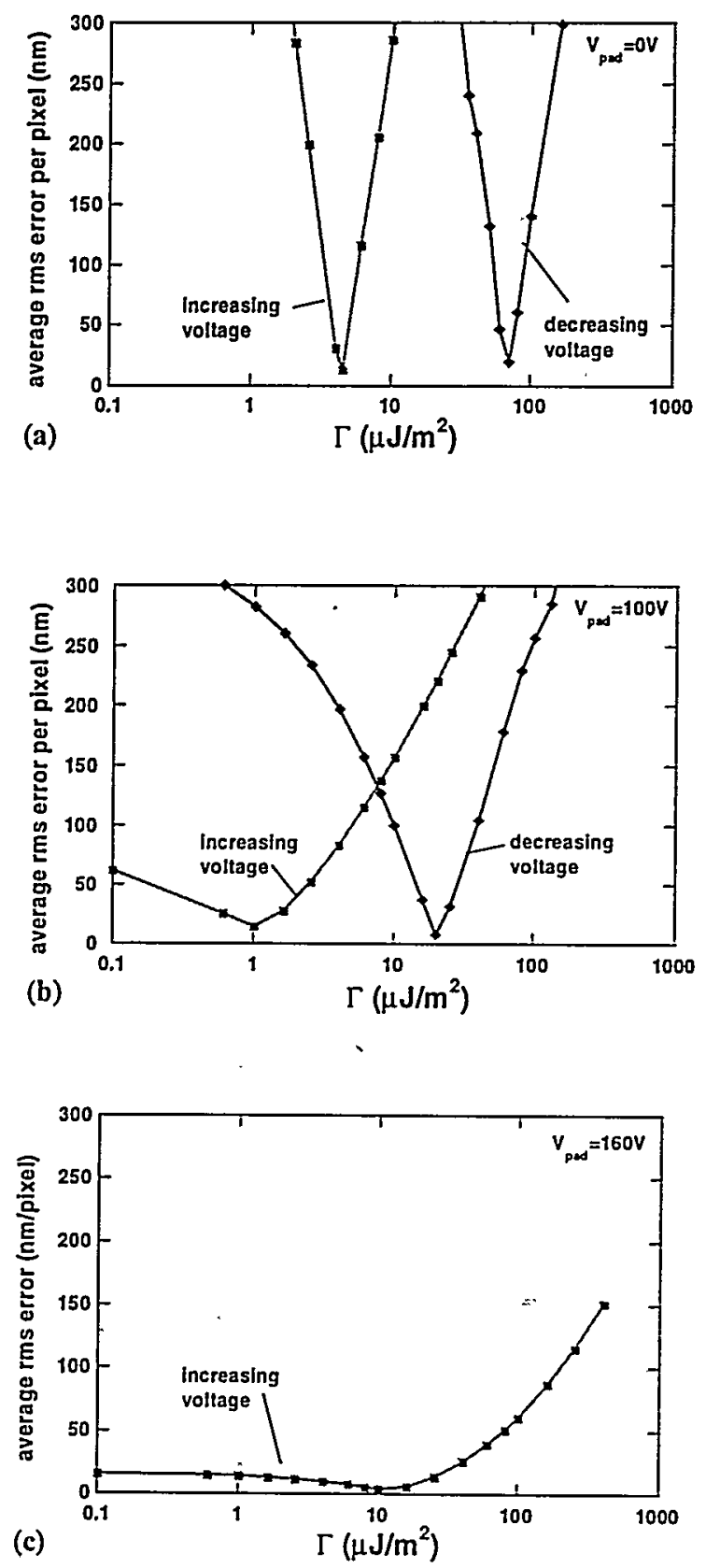

Fig. 10 Average rms per pixel curves versus adhesion at applied voltages of (a) $0 \mathrm{~V}$, (b) $100 \mathrm{~V}$ and (c) $160 \mathrm{~V}$. The adhesion is taken as the minimum in each curve. 

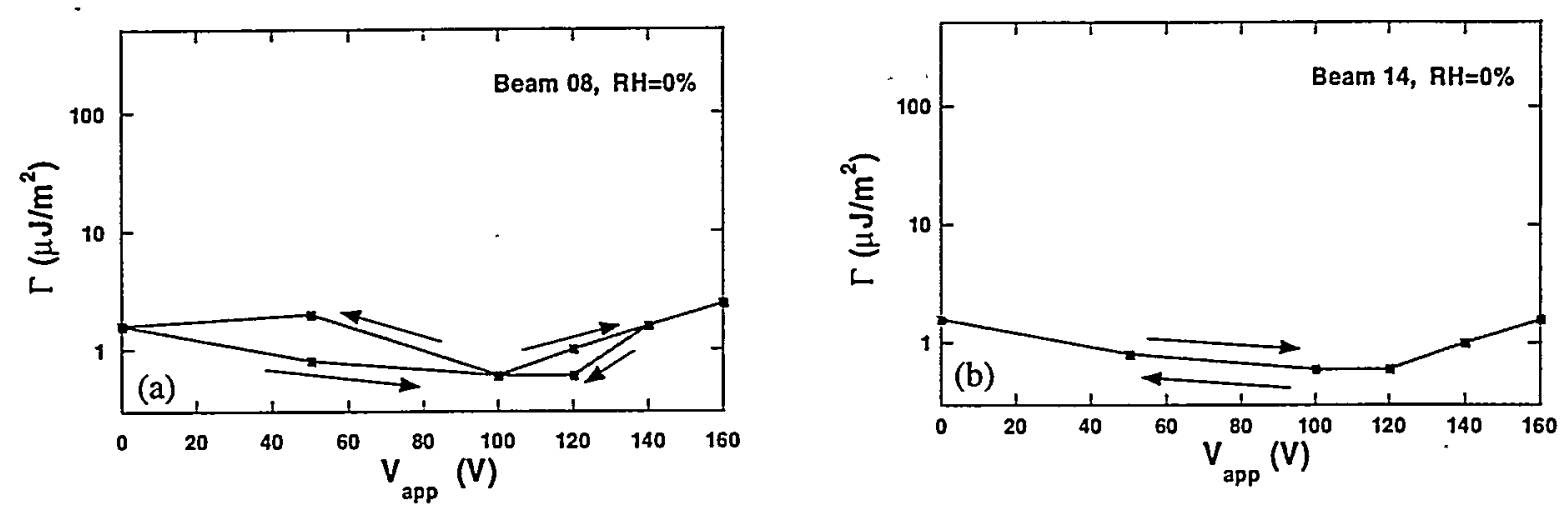

Fig. 11 Adheșion vs. voltage for two beams at $\mathrm{RH}=0$.
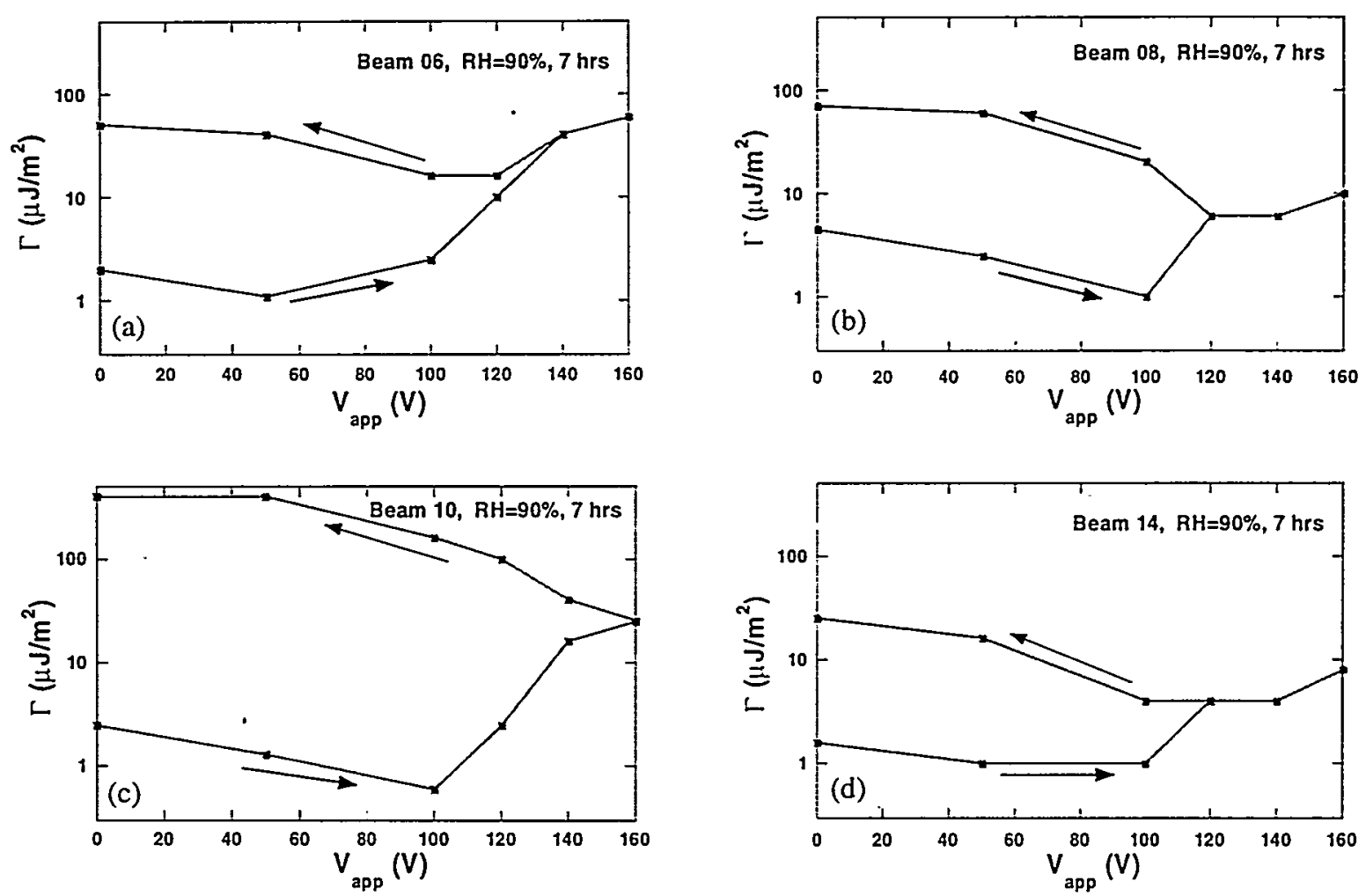

Fig. 12 Adhesion vs. voltage for four beams at RH=90\%, 7 hour exposure. (the beam numbers are counted from the top of the cantilever array of Fig. 8) 


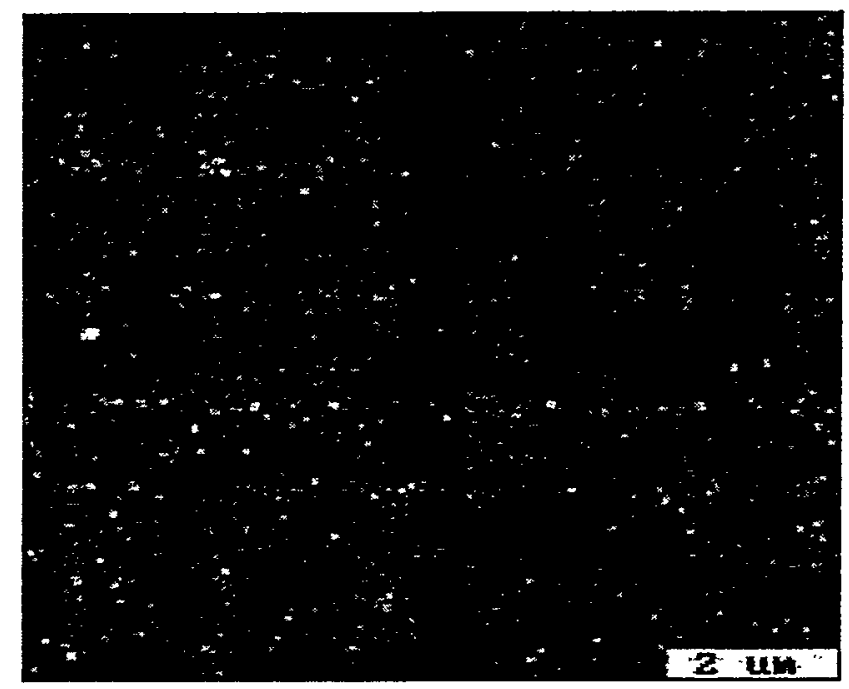

Fig. 13(a) Landing pad roughness at low RH

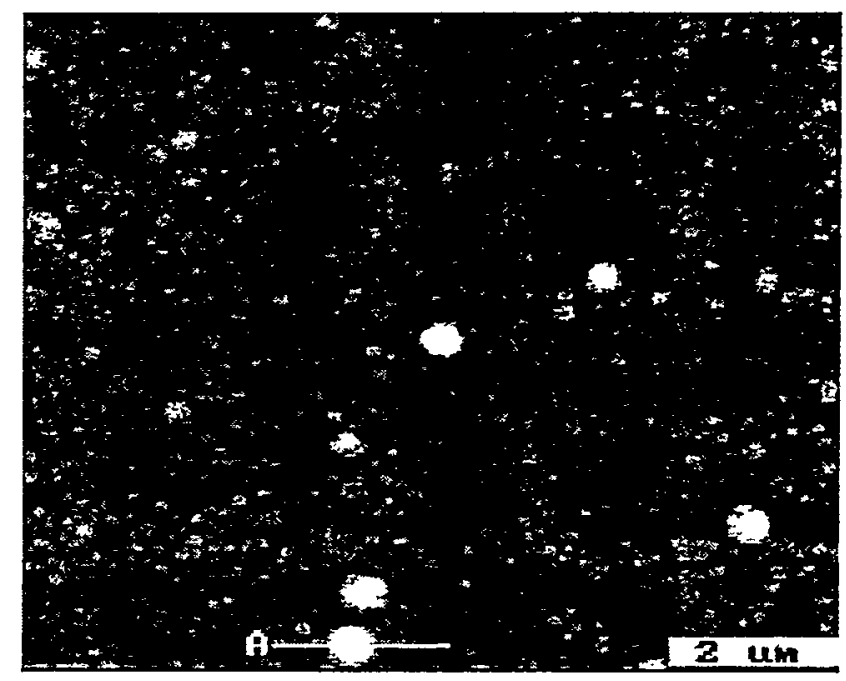

Fig. 13(b) agglomerated mounds of FDTS after exposure to high RH.

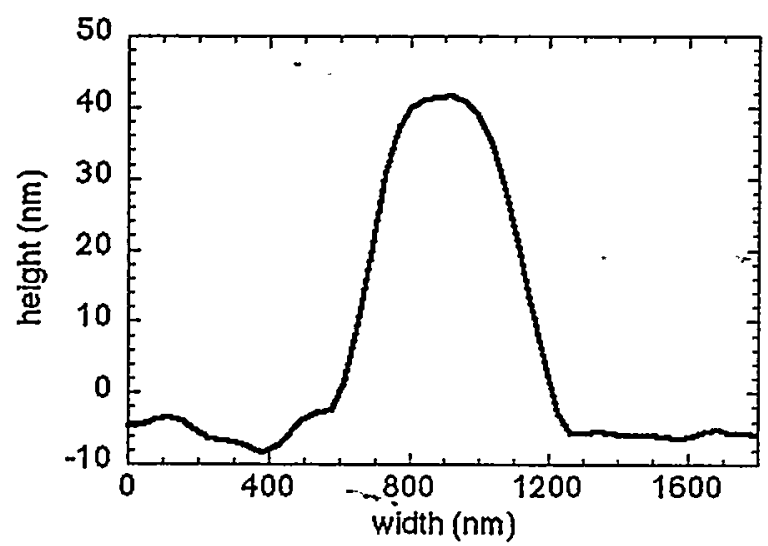

Fig. 13(c) linescan across a large mound. 


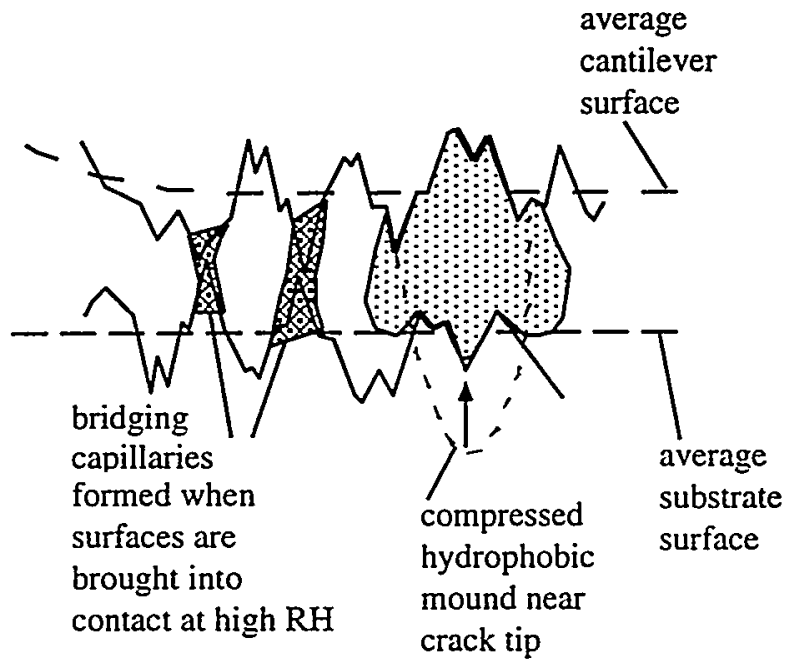

Fig. 14 Soft protruding areas compressed at the crack tip under large externally applied loads allow nearby hydrophilic areas to come into contact. 